\title{
Atmospheric effects on cratering on Venus
}

\author{
T. Takata ${ }^{1}$ and T. J. Ahrens \\ Division of Geological and Planetary Sciences, California Institute of Technology, Pasadena \\ R. J. Phillips \\ Department of Earth and Planetary Sciences, Washington University, Saint Louis, Missouri
}

\begin{abstract}
A paraboloidal bow shock model is developed in order to estimate the surface distribution of gas shock-induced modifications surrounding Venusian impact craters. We apply two-dimensional oblique shock dynamics to describe a three-dimensional paraboloidal-shaped bow shock impinging upon an assumed incompressible Venusian surface. The effects of the hypersonic atmospheric shock acting on the Venusian surface are considered in terms of induced maximum gas pressure, density, particle velocity, and temperature, for varying angles and velocities of impact. The maximum boulder size that can be saltated by the shock wave induced gas flow and the degree of mutual collision of the surface materials are also considered. The present calculations quantitatively predict the areal extent of the gas shock perturbed surface for normal and oblique impact as a function of impact angle and velocity, and radii of impactors. For a $1-\mathrm{km}$ radius stony meteorite impacting normally at $20 \mathrm{~km} / \mathrm{s}$, the radius of the disturbed area extends $\sim 10-17$ times the $3-5 \mathrm{~km}$ crater radius. The perturbed surface affects the surface radar properties, and the present results can provide an explanation of the wide "dark/bright halos" surrounding some of the Venusian impact craters observed via Magellan imagery. For example, a $\sim 50$-km radius bright halo surrounding a $\sim 20-\mathrm{km}$ dark halo is observed around the $3.1-\mathrm{km}$ radius crater located at $16.5^{\circ}$ north latitude and $334.4^{\circ}$ longitude. The average value of the radar backscatter cross section of the $\sim 20-\mathrm{km}$ radius dark halo indicates that $\sim 50$-cm-thick layer of porous lithologic material is superimposed upon an assumed undisturbed basement rock surface. The bright halo indicates that the surface roughness in this region is $\sim 30 \%$ greater than that of the surrounding original surface. These features can be induced by atmospheric shock waves. The present model can relate the observed crater halo radii to the impact parameters, such as projectile radius and density, and the impact velocity and angle.
\end{abstract}

\section{Introduction}

Venus has a dense atmosphere, whose pressure and density on the surface are $9.2 \mathrm{MPa}$ and $65.0 \mathrm{~kg} / \mathrm{m}^{3}$, respectively [Serff, 1983]. Among the terrestrial planets, this dense atmosphere is unique to Venus. Meteoroids upon transversing this atmosphere are affected by the resulting deceleration, ablation, deformation, and fragmentation. The atmosphere also affects impact ejecta emplacement and reduces the final crater dimensions relative to atmosphere-free planets [Melosh, 1989]. If there is no significant deceleration, incident

\footnotetext{
${ }^{1}$ Now at Geological Institute, University of Tokyo, Tokyo, Japan.
}

Copyright 1995 by the American Geophysical Union.

Paper number 95JE02641.

0148-0227/95/95JE-02641\$05.00 meteoroids would impact the Venusian surface with hypersonic velocity. During atmospheric transit, an atmospheric shock front forms. The shock wave driven by the meteoroid impinges on the Venusian surface. As a result, features normally associated with impact cratering on atmosphere-free planets are modified in the case of impact cratering on Venus. Upon interpreting the Venera radar imaging, Ivanov et al. [1986] first estimated the effect of the gas shock. They explained qualitatively and approximately estimated the magnitude of shock interactions with the surface using the results of analog high-explosive experiments.

Magellan radar backscatter data revealed that some craters are surrounding by "dark halos", which have low radar backscatter cross sections, and that these features are unique to Venus [Phillips et al., 1991] (Figure 1). The halo in Figure 1d has no crater, but still has the same features as other craters. Some of the dark halos are themselves accompanied by surrounding bright halos, as in Figures $1 \mathrm{a}, \mathrm{b}$, and $\mathrm{d}$. In the case of oblique 

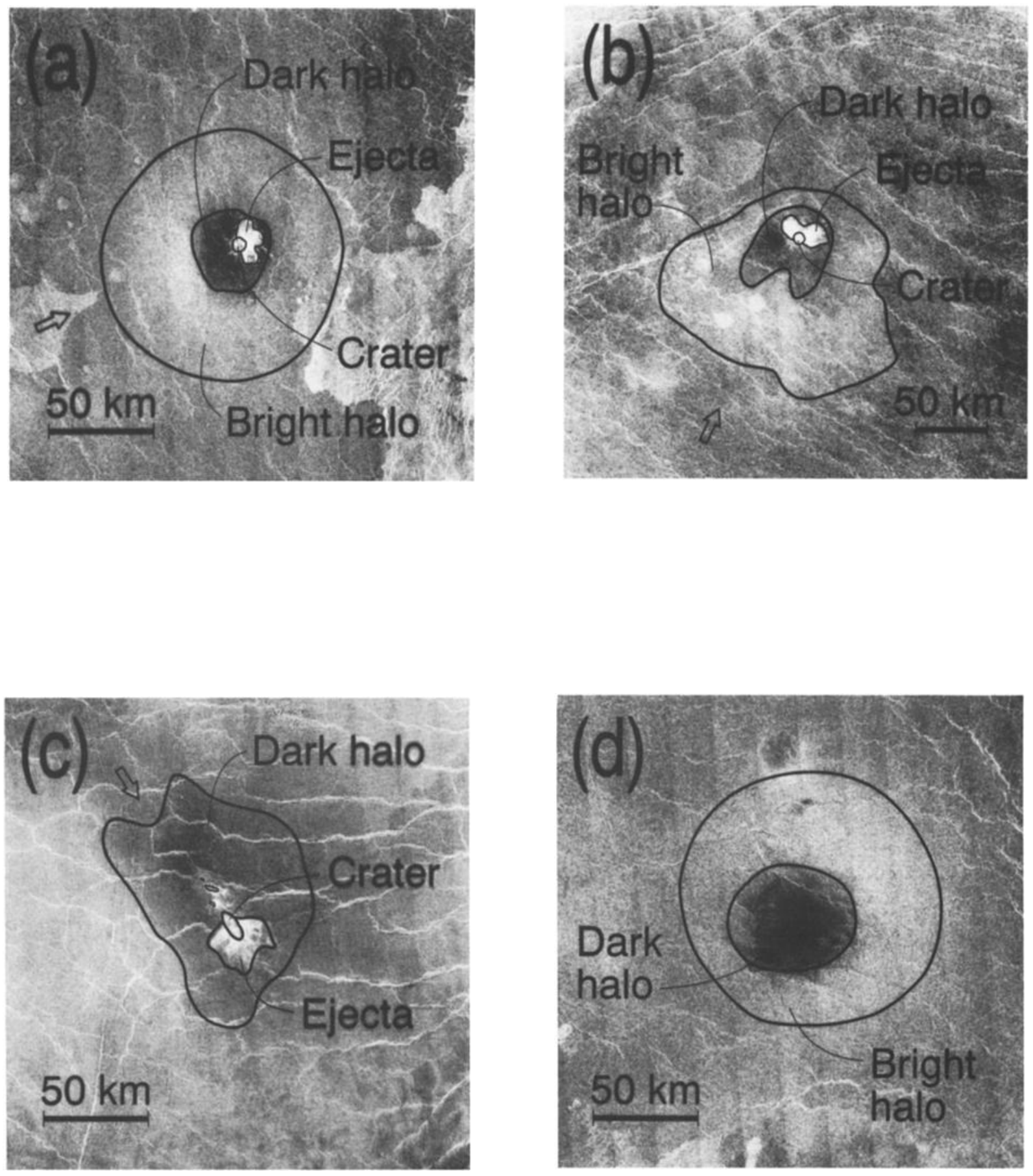

Figure 1. Magellan images of craters that have wide dark or bright halo features. (a) Unnamed crater, mean diameter $6.1 \mathrm{~km}$, at $16.5^{\circ} \mathrm{N}$ and $334.4^{\circ} \mathrm{E}$. (b) Unnamed crater, mean diameter 8.0 $\mathrm{km}$, at $11.9^{\circ} \mathrm{N}$ and $352^{\circ} \mathrm{E}$. (c) Unnamed crater, mean diameter $11 \mathrm{~km}$, at $9.3^{\circ} \mathrm{N}$ and $358^{\circ} \mathrm{E}$. (d) Bright and dark halo without crater, at $8.7^{\circ} \mathrm{N}$ and $333.5^{\circ} \mathrm{E}$.

impact, the dark halos seem to elongate up-range from the impact craters, whereas the continuous ejecta pattern extends preferentially down-range. The variation of the backscatter cross section at constant radar incident angle around craters is explained by either the difference of the surface roughness, variation in the porosity of the surface materials, or variation in lithology. A region of lower backscatter cross section, compared to the surrounding region, will occur when the surface is smoother than its surroundings. Another interpretation is that it is more porous than the original planetary surface. In contrast, an area with brighter backscatter cross sections indicates that surface is rough at the scale of the Magellan radar wavelength $(12.6 \mathrm{~cm})$. The Pioneer Venus radar reflectivity data showed that a smooth appearing surface is dominated by bedrock or densely 
packed debris, and that the thickness of a fine-grained regolith cover should be less than tens of centimeters thick [Arvidson et al., 1991]. We also note that Venera 13 and 14 landing sites imaging data showed slablike rocks and rock fragments with dimensions of several centimeters to several tens of centimeters [Florenskiy et al., 1983]. Therefore the modification of the radar properties as a result of impact cratering must overprint these features of the original surface.

In the case of the crater shown in Figure la, the median value of the backscatter cross section, $\sigma_{\text {med }}$, of Figure $1 \mathrm{a}$ is $-14.2 \mathrm{~dB}$, that of the region of the dark halo, $\sigma_{D}$, is $-17.6 \mathrm{~dB}$, and that of the region of bright halo, $\sigma_{B}$, is $-11.6 \mathrm{~dB}$ as measured along the longest radius of the halo of this crater. The backscatter cross section is obtained by the conversion from the pixel value in synthetic aperture radar (SAR) image data, which is normalized by the scattering coefficient of Muhleman's law for the radar incidence angle $\left(\sim 44^{\circ}\right.$ at $\left.16.5^{\circ} \mathrm{N}\right)$ [Hyon, 1991; J. J. Plaut, personal communication, 1991].

First, we assume that the dielectric constant of the surface materials and the radar incidence angle are the same everywhere in this region, that the surface is flat, and that the change in backscatter cross section is caused mainly by the change of surface roughness, $h$. The difference $(\Delta)$ in $h$ is related to the difference in backscatter cross section (in $\mathrm{dB}$ ) by

$$
\Delta[\log (h)]=0.05 \Delta \sigma
$$

for Bragg scattering [Ulaby, 1982]. For (1) to be valid, $h$ must be less than the scale of radar wavelength $(\lambda)$. From this relationship, the average values of relative ratio of roughness of the disturbed regions (dark halo), $h_{D}$, to the original surface, $h, h_{D} / h$, and that of the bright halo, $h_{B}$, to the original surface, $h_{B} / h$, of the crater in Figure 1a are approximately 0.7 and 1.3, respectively.

Alternatively, we could assume that the change of backscatter cross sections is caused by the radar penetration into a surface layer of varying degree of porosity. The relationship of the difference of the layer thickness $\Delta d$ and the difference of the backscatter cross section $\Delta \sigma \mathrm{dB}$ can be expressed approximately,

$$
\Delta d=-0.12 L_{p} \Delta \sigma
$$

where $L_{p}$ is the penetration depth, which is $9.2 \lambda$ for dry soil [Elachi, 1987]. From this equation, the region of dark halo has an $\sim 50 \mathrm{~cm}$ thicker porous surface layer superimposed on the original average surface materials. Assuming that the high backscatter cross section is caused by the lack of a porous layer, the thickness of the porous layer of the region of the bright halo is approximately $35 \mathrm{~cm}$ thinner than that of the original surface. If the original surface feature of the impact site is similar to the lithology of the Venera landing site, the porous layer is not thick enough to be excavated more than $30 \mathrm{~cm}$.

Phillips et al. [1991] suggested that "dark halos" originate from either the effect of impact of the atmospheric shock wave that trails the meteoroid, or represent a deposit of the fine material produced by the ablation of the meteoroid in the atmosphere. Zahnle [1992] assumed small bolides exploded in the atmosphere and approximated the expansion of the atmospheric shock wave with a spherical blast wave model. In his model, dark and bright halos are explained by the difference of sizes of the broken rocks. The effect of an atmospheric shock on the surface is also investigated by Ivanov et al. [1992].

We develop a bow shock model in order to quantify the effects of the hypersonic atmospheric shock accompanying oblique impact on the planetary surface. The related flow properties, such as maximum gas pressure and gas tangential shear flow velocity, are calculated. Oblique shock dynamics are applied to the threedimensional paraboloidal bow shock front interacting with an incompressible rigid half-space to understand the interaction of the gas shock with the planetary surface. Then the magnitude of the surface disturbance is estimated from the model. We describe how the bow shock model applies to interpreting the radar data of impact craters which have bright and dark halos surrounding impact craters. It is also investigated how halo size may be used to estimate impact parameters associated with a given crater.

\section{Bow Shock Model and Shock Physics}

\subsection{Bow Shock Formation}

When a hypervelocity meteoroid encounters an atmosphere, a shock front forms around the meteoroid and induces a compressed gas layer between the shock front and the meteoroid [Bronshten, 1983] (Figure 2a). In addition, a bow shock wave and a wake develop behind the body and expand toward the incoming direction [Martın, 1966].

In the case of a meteoroid passing through an atmosphere with high Mach number $M$ (ratio of shock velocity to local atmospheric sound speed) and with the corresponding low value of $\gamma$ (the ratio of the specific heat at constant pressure relative to that at constant volume), the compressed gas layer becomes very narrow compared to the size of the body. Assuming that the body is a sphere, and that $M>2$, the thickness, $\Delta_{s}$, of the compressed layer, that is, the distance between the shock front and the body at the stagnation point is expressed as

$$
\Delta_{s} / R \sim 2 /\left[3\left(\rho / \rho_{0}-1\right)\right] \sim(\gamma-1) / 3
$$

where $R$ is the radius of the body, $\rho$ is the atmospheric density of the shocked state, and $\rho_{0}$ is the atmospheric density of the ambient state [Martin, 1966, equation (5-2)]. $\Delta_{s} / R$ becomes 0.07 for $\gamma=1.2$, and 0.03 for $\gamma=1$.1. Since the effective value of $\gamma$ tends to unity at higher $M, \Delta_{s} / R$ approaches zero and the shock front gets close and tangent to the body at the stagnation point, when $M \gg 1$ [Hayes and Probstein, 1966, p. $420]$. 

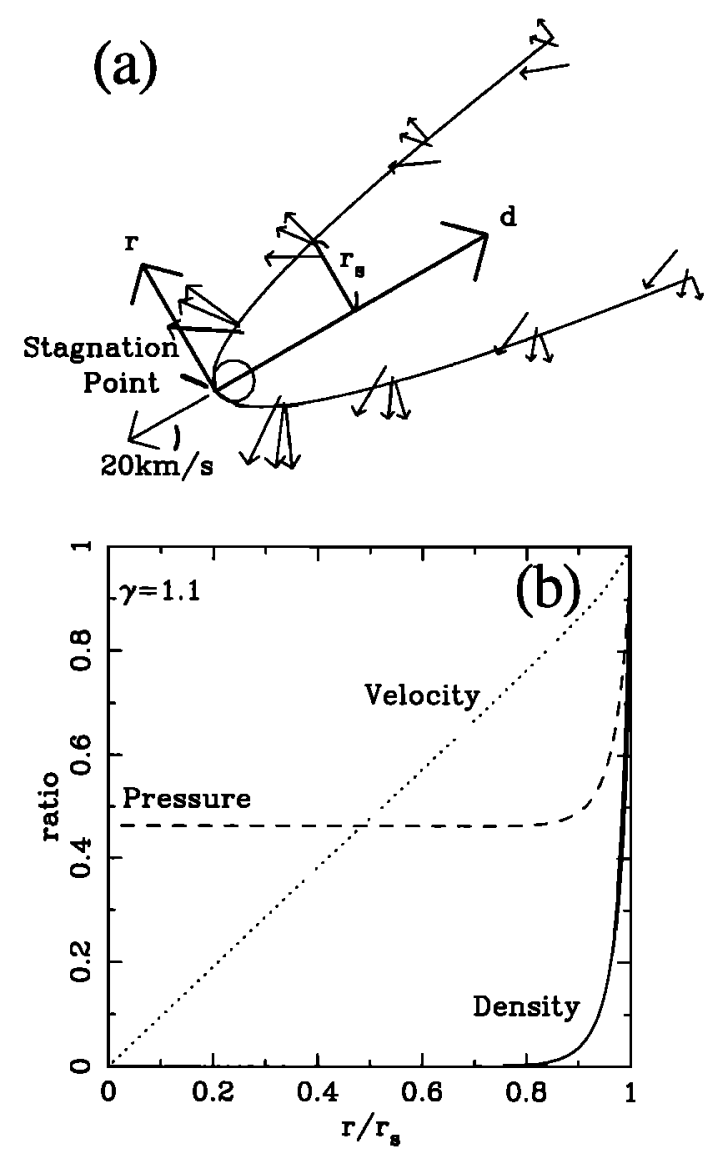

Figure 2. (a) The bow shock shape calculated from the symmetric power law (equation (4)) and corresponding gas flow velocity. Here, $r$ is the distance of the bow shock front from the trajectory of the meteoroid and $d$ is the distance along the trajectory from the tip of the meteoroid. The meteoroid is traveling at a velocity of $20 \mathrm{~km} / \mathrm{s}$ in this case. The arrows represent the velocity of the gas at each position of the space in the reference frame of the stationary planetary surface. (b) Shock parameters, such as atmospheric density, pressure, and gas velocity inside the bow shock, normalized by these shock parameters at the shock front, $r_{s}$, calculated from the symmetric power law, (A1)-(A3), in the case of steady flow.

Thus the shock front has the same local curvature as the meteoroid on the stagnation point, and the shape of the bow shock wave approaches a paraboloidal surface. The normal distance of the bow shock wave from the trajectory of the meteoroid $r$ is a function of the distance from the tip of the body along the trajectory $d$ (as shown in Figure 2a [Martın, 1966]):

$$
r / R \sim(2 d / R)^{\frac{1}{2}}
$$

The gas flow behind the bow shock can be calculated using a similarity solution for an inviscid axisymmetric expanding wake [Zel'dovich and Razzer, 1966]. This is shown in Figure 2a [Hornung, 1967]. In Figure 2a, the meteoroid is moving at $20 \mathrm{~km} / \mathrm{s}$. The arrows represent the particle velocity in the gas flow in the reference frame of the stationary planetary surface. The ratio of the gas velocity, pressure, and density at the wake position $(r)$ relative to those right behind the shock front $\left(r_{s}\right)$ at the same travel distance from the stagnation point $(d), v / v_{s}, p / p_{s}$, and $\rho / \rho_{s}$, respectively, are calculated as a function of normalized wake distance $r / r_{s}$ (Figure 2b). Here, the subscript $s$ indicates the value behind the shock front. In this calculation, $\gamma=1.1$ is assumed. The gas is compressed toward the region close to the shock front (Figure 2b). If $\gamma$ increases, the pressure and density curves, such as those in Figure $2 b$, fall off less rapidly.

\subsection{Bow Shock Model}

Assuming that the body retains the same spherical curvature at the front during its traverse through the atmosphere, the meteoroid is accompanied by a bow shock wave whose shape remains constant and is given by (4). The particle velocity of the gas at the shock front is normal to the shock front, as shown in Figure 2a. The gas enveloped by the shock subsequently expands forward and away from the trail of the meteoroid. The paraboloidal bow shock continues to propagate away from the trajectory, and it interacts with the planetary surface, as sketched in Figure 3a, assuming a uniform atmosphere. In the reference frame of the surface at rest, the shock wave propagates away from the center of the crater. The propagation and reflection of the shock wave at around the contact point of shock wave on the planetary surface is analogous to the case of spherically expanding blast wave interacting with the surface [Hellig, 1985; Brode, 1968] (Figure 3c). However, in the case of oblique impacts, the trajectory of the blast shock wave trailing the meteoroid is also inclined toward the surface, and the blast wave interacts with the up-range surface at an earlier point in time than the impact of the meteoroid (Figure $3 \mathrm{~b}$ ). Therefore a different interaction from that of a spherical explosion is expected, and a paraboloidal bow shock provides a framework for describing the interaction of the atmospheric shock with planetary surface in the case of oblique impact.

To quantify the position of the paraboloidal bow shock front as a function of time, we employ a coordinate system to define the surface position, $X$ and $Y$, and vertical elevation, $Z$. We assume that the meteoroid trajectory lies in the $X-Z$ plane. Here $\Phi$ is the angle of the trajectory from the horizon (Figure 4a). The unit of length is normalized to the radius of a meteoroid, $R$. We express the bow shock surface as $f=0$, where $f$ is

$$
\begin{aligned}
f=\quad & \left(\frac{Y}{R}\right)^{2}+1\left(\cos \Phi \frac{Z}{R}-\sin \Phi \frac{X}{R}\right)^{2} \\
& -\left[2\left(\cos \Phi \frac{X}{R}+\sin \Phi \frac{Z}{R}-\frac{X_{0}}{R}\right)\right]^{2 n}
\end{aligned}
$$

Here, $X_{0}=X_{r}-V_{\imath} t, X_{r}$ is an arbitrary reference distance at $t=0, V_{i}$ is the impact speed of the meteoroid, $\mathbf{V}_{i}=\left(-V_{i} \cos \Phi, 0,-V_{\imath} \sin \Phi\right)$, and $n=\frac{1}{2}$ for a paraboloid. 

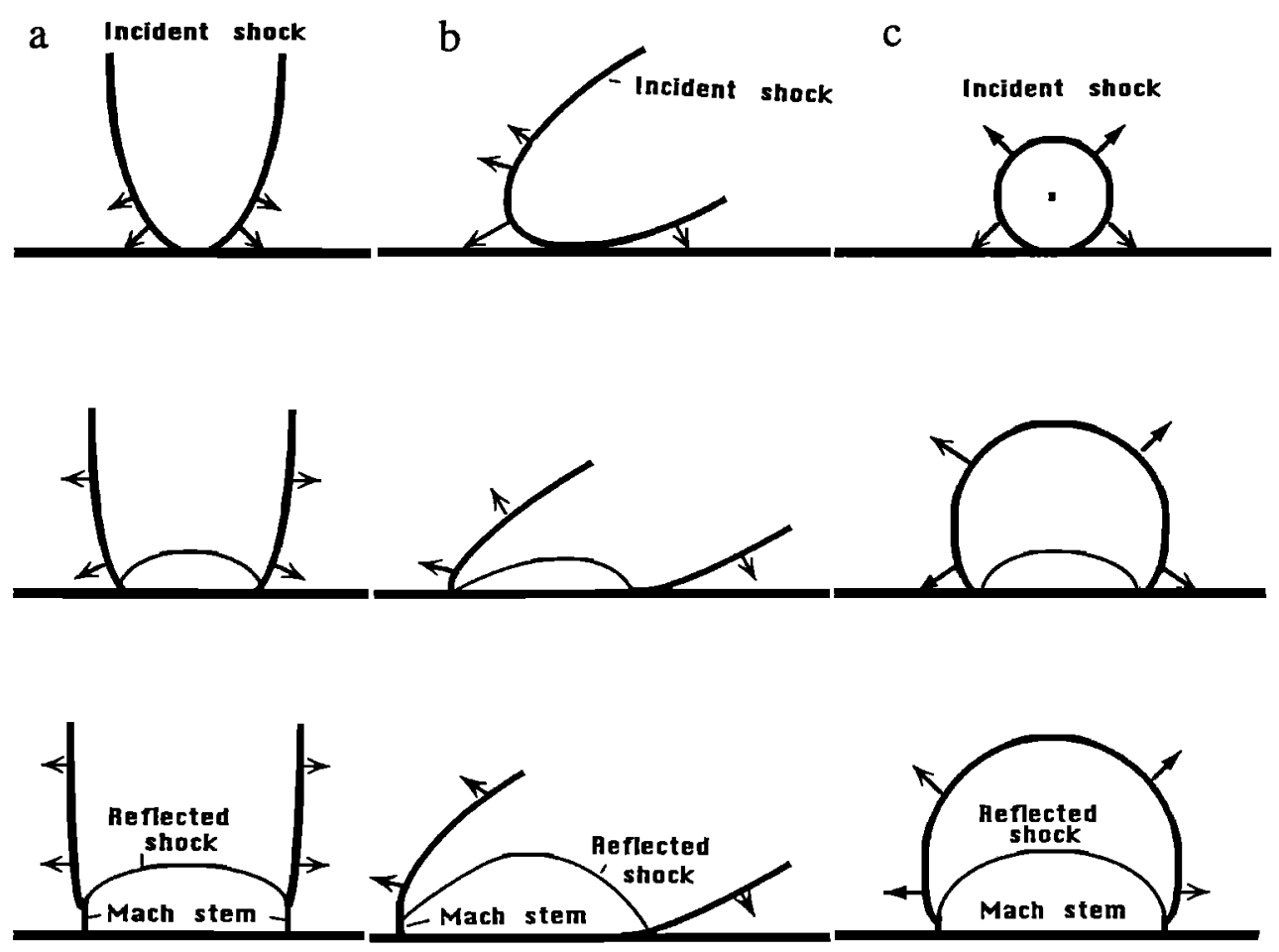

Figure 3. Sketch of propagation and interaction with the planetary surface of (a) a bow shock wave for normal impact, (b) a bow shock wave for oblique impact, and (c) for comparison, a spherical blast wave from an atmospheric explosion.

For some values of $\Phi,(5 \mathrm{a})$ cannot be applied for $X \leq 0$, since the shock front expressed by this equation at these points is inclined toward the incoming direction on the planetary surface, and thus it is no longer appropriate for the propagating shock. However, when the meteoroid impacts the surface, the atmospheric gas is compressed between a meteoroid and the surface. The compressed atmospheric gas on the impact site can be explosively released cylindrically [O'Keefe and Ahrens, 1988]. Therefore, we assume that the shock front propagates cylindrically along the planetary surface in the down-range direction in the same way as the case of normal impact. Then, for the oblique impact, instead of (5a), in the region of $X<0$ and $-X / Z<\tan \Phi$, we use

$$
f=\left(\frac{Y}{R}\right)^{2}+\left(\frac{X}{R}\right)^{2}-\left[2\left(\frac{Z}{R}-\frac{X_{0}}{R}\right)\right]^{2 n}
$$

Then the propagation of the shock wave on the surface is similar to that of normal impact $(Z=0$ in $(5 \mathrm{~b})$ corresponds to $Z=0$ and $\Phi=90^{\circ}$ in (5a)), and (5a) and (5b) are continuous at $X / Z=\tan \Phi$. Figure 4 shows the time varying position of the propagating bow shock front in a vertical cross section and ground foot print, in the case of $\Phi=30^{\circ}$, with time intervals equal to $100 R / V_{\imath}$. The position of the bow shock front in a vertical cross section is expressed as $f(t, Y=0)=0$ (Figure 4a), and the position of the shock front on the surface (Figure $4 \mathrm{~b})$ can be expressed as $f(t, Z=0)=0$ in (5a) and (5b). The shock fronts defined by $(5 \mathrm{~b})$ are plotted with dotted lines in Figure $4 \mathrm{a}$.
The horizontal propagation velocity of the shock front on the planetary surface, $q_{1}$, Mach number of $M_{1}$, and shock angle, that is, the inclination angle of the shock front from the ground surface, $\alpha$, are calculated from (5a) and (5b) at each point on the planetary surface as

$$
q_{1}=c_{1} M_{1}=\frac{1}{\sin \alpha}\left(\mathbf{V}_{\imath} \cdot \frac{\nabla f}{|\nabla f|}\right)
$$

and

$$
\sin \alpha=\sqrt{f_{x}^{2}+f_{y}^{2}}
$$

where $c_{1}$ is the sound speed of the ambient state and we define $\nabla f /|\nabla f|=\left(f_{x}, f_{y}, f_{z}\right)$.

To compare the paraboloidal bow shock to cylindrical and spherical atmospheric blast wave, we will investigate the position of the propagating shock wave for these cases. In the case of the normal impact of the meteoroid $\left(\Phi=90^{\circ}\right)$, the distance of the shock front on the planetary surface $(\mathrm{Z}=0)$ from the center of the impact as a function of time, $r_{b}(t)$, can be written as

$$
\frac{r_{b}(t)}{R}=k_{b}\left(\frac{V_{\imath} t}{R}\right)^{\frac{1}{2}}
$$

where $k_{b}$ is $\sqrt{2}$.

In the case of a strong spherical blast wave, the distance of the shock front from the point of the explosion, $r_{s p}(t)$, becomes

$$
\frac{r_{s p}(t)}{R}=k\left(\frac{E}{\rho}\right)^{\frac{1}{5}} t^{\frac{2}{5}} \sim k_{s}\left(\frac{V_{i} t}{R}\right)^{\frac{2}{5}}
$$



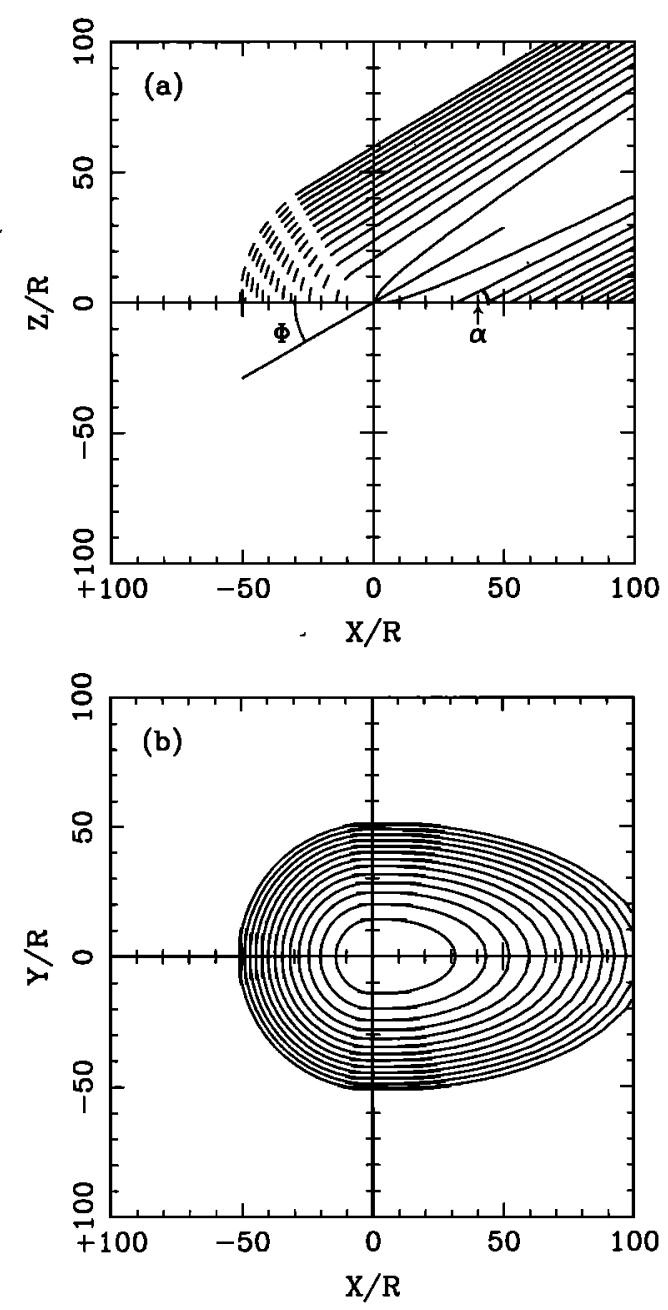

Figure 4. Time varying position of propagating bow shock front (a) vertical cross section, (b) footprint on the ground, for $\Phi=30^{\circ}$ with a time interval of normalized time of $100 R / V_{2}$. In equations (5a) and (5b), the condition of $Y=0$ corresponds to the propagating blast wave in Figure $4 \mathrm{a}$. The dotted line expresses the position applied by (5b). The coordinate is normalized by the meteoroid radıus $R$, and the center of the meteoroid passes through the origin of the coordinate system.

where $k$ is a coefficient, equal to approximately unity ( 0.8 for $\gamma=1.1$ and 0.9 for $\gamma=1.2$ ) and $E$ is the total energy of the explosion [Zel'dovich and Rauzer, 1966, p. 99]. Here we use the kinetic energy of the meteoroid for the explosive energy $E$ with the coefficient of the efficiency of the conversion from the kinetic energy of the meteoroid to the explosive energy of the gas, $\varepsilon(\leq 1)$, where $E=(\varepsilon / 2)(4 \pi / 3) R^{3} \rho_{m} V_{\imath}{ }^{2}, \rho_{m}$ is the density of the meteoroid, and $k_{s}$ is the coefficient of $\simeq k\left(2 \varepsilon \rho_{m} / \rho\right)^{\frac{1}{5}}$. The value of $\varepsilon$ depends on impact parameters and is not well determined; however, $O^{\prime} K$ Keefe and Ahrens [1993] demonstrated that $\varepsilon$ is 1 to 0.1 for meteoroids of $10^{2} \mathrm{~m}$ to $1 \mathrm{~km}$. Therefore the difference of $\varepsilon$ only affects $k_{s}$ by less than a factor of 10 . In the case of a cylindrical blast wave, the distance of the shock front from the explosive point, $r_{c}(t)$, becomes

$$
\frac{r_{c}(t)}{R}=\sqrt{2} k^{\prime}\left(\frac{E}{\rho}\right)^{\frac{1}{4}} t^{\frac{1}{2}} \sim k_{c}\left(\frac{V_{c} t}{R}\right)^{\frac{1}{2}}
$$

where $k^{\prime}$ is another coefficient whose magnitude is approximate unity [Sakuraı, 1968, p. 348]. Here, $k_{c}$ becomes $\sim \mathrm{R}^{1 / 4}$ at $\varepsilon=0.01$ and $\sim 4 R^{\frac{1}{4}}$ at $\varepsilon=1$, assuming that $\rho_{m}=2700 \mathrm{~kg} / \mathrm{m}^{3}$.

The ratio between the radius of the paraboloidal bow shock propagating outward and that of the spherical explosion is

$$
r_{b}(t) / r_{s p}(t) \sim\left(k_{b} / k_{s}\right)\left(V_{i} t / R\right)^{-01}
$$

Since $k_{b} / k_{s}$ is approximately unity, the difference of the two models depends in detail on impact parameters. Similarly, the ratio between the radii of the propagating bow shock and that of the cylindrical explosion is described by

$$
r_{b}(t) / r_{c}(t) \sim k_{b} / k_{c} \sim R^{-\frac{1}{4}}
$$

(12) shows that these ratios are of the order of unity, that is, the paraboloidal bow shock is analogous to a cylindrical blast wave. Therefore the paraboloidal bow shock model can be treated as the cylindrical blast wave, by taking into account oblique impact.

\subsection{Reflected Shock Wave}

Next, we will examine the atmospheric shock state via the interaction with the surface. In the case of a strong surface explosion (e.g., nuclear), a thermal precursor in the atmosphere occurs because underground shock initially outruns the atmospheric shock, and subsequently preheats the atmosphere immediately above the ground. The main atmospheric shock is also reflected from the surface, and the reflected shock is also transmitted backward from the incident shock front on the ground surface [Brode, 1968, Figure 15]. The nature of the gas shock reflection also changes as the atmospheric shock front propagates away from the center of the explosion. The effect of the shock reflection following the incident shock is comparable to the incident shock. For example, the ratio of the pressure behind the reflected shock to the pressure in front of the reflected shock (or behind the incident shock), $p_{3} / p_{2}$, is $\sim(3 \gamma-1) /(\gamma-1)$ in the case of a tangential shock when the ratio of the pressure behind the incident shock to that of the ambient pressure is $p_{2} / p_{1} \gg 1$. Then $p_{3} / p_{2}$ becomes 13 for $\gamma=1.2$, and 23 for $\gamma=1.1$ [Courant and Friedruchs, 1948, p. 153]. Here, subscript 1 indicates the ambient state, and subscript 2 indicates the pressure behind the incident shock, whereas subscript 3 indicates the pressure behind the reflected shock (Figure $5)$. Thus more than one order of magnitude difference can be observed in the pressure behind the reflection shock, and it is essential to take into account the effects of shock reflection, in order to estimate the actual shock properties at the contact points of shock wave on the planetary surface and the magnitude of the disturbance on the surface. The effect of enhanced pressure by shock reflection wave was discussed by Brackett and $M c K i n$ non [1992]. However, geometrical application of oblique impacts has not been previously taken into account. 
(a)

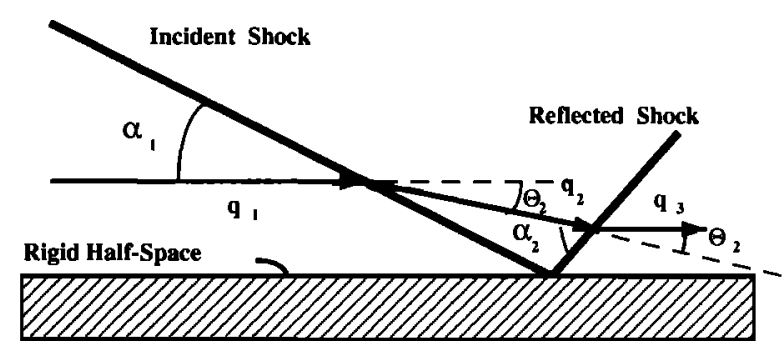

(b)

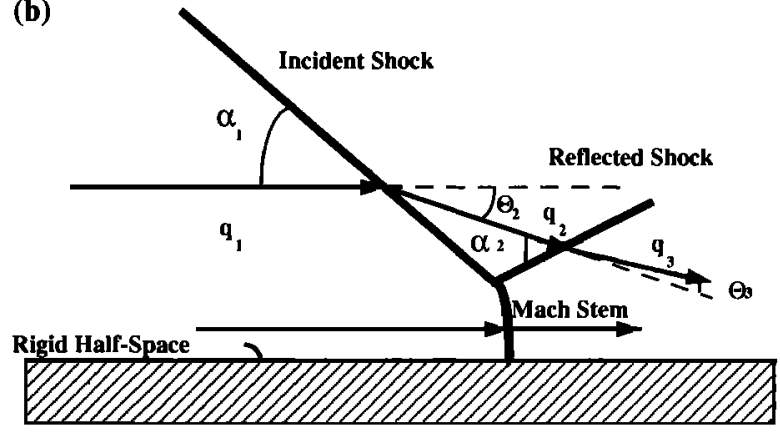

Figure 5. Sketch of (a) regular reflection and (b) Mach reflection. (a) The initial gas flow and final flow are the same direction in the case of regular reflection. The intersection of the initial and reflected shock front is on an assumed rigid planetary surface. (b) Impact angle $\alpha$ is large enough for the intersection of incident shock front and reflected front to detach as Mach stem from the ground [data from Hornung, 1986].

For constant $\gamma$, the configuration of the reflected shock and the state behind the shock (pressure, density, temperature, and particle velocity) can be estimated from the horizontal component of the shock front propagation velocity, $q_{1}$, in (6), and from the angle of the shock front, $\alpha$, using the conservation equations for oblique shocks (Appendix B).

We summarize the configurations of shock reflection in Figure 5 [from Hornung, 1986]. When the value of the properties within two of the shocked regions (as depicted in Figure 5) are the same, we use the lowest value of appropriate subscript. Here, the velocity of the gas flow is expressed in the frame of reference of the stationary shock front. When $M_{1}$ is less than $(\sin \alpha)^{-1}$, a shock does not form and the flow becomes subsonic, and when the gas velocity behind the incident shock $M_{2}\left(=q_{2} / c_{2}\right)$ is $<1$, the reflected shock is diffusive, and the inclination of the incident shock front becomes gradually perpendicular to the surface (we denote this case as "no reflection").

Two other types of shock reflection exist. Regular reflection is shown in Figure 5a. Mach reflection is shown in Figure 5b. In the latter case, the intersection of the incident and reflected shock does not contact the planetary surface, and a Mach stem is developed [Hornung, 1986].

The calculated shock reflection types, as a function of $\alpha$ and $M_{1}$ in the case of $\gamma=1.1$, are shown in Figure 6a. The regions are divided by the types of no reflection (diffusive reflection), Mach reflection, regular reflection, and subsonic region (unhatched region in the figure). In order to see the effect of varying the value of $\gamma$, we plotted the post-shock pressure for $\gamma=1.1$ (solid line) and $\gamma=1.2$ (dotted line) in Figure 6b. The discontinuity of the isobar around $\alpha \sim 45^{\circ}$ in this figure is caused by the change of the reflection type (the boundary between regular reflection and Mach reflection). If $\gamma$ becomes smaller, the regular reflection occurs at greater $\alpha$. If the type of reflection is regular, the difference of the pressure between $\gamma=1.1$ and $\gamma=1.2$ becomes about a factor of 2 at high Mach numbers. We will discuss the selection of the effective $\gamma$ for shocked $\mathrm{CO}_{2}$ gas in the next section.

In these calculations, we assume that the flow of the gas in front of and behind the shock front is pseudosteady inviscid flow of a perfect gas in the thermal equilibrium. Pseudo-steady flow means that the shock wave is assumed to propagate into a stationary gas in an inertial frame with the speed of $q_{s}\left(=q_{1} \sin \alpha\right)$. This can be treated as the steady horizontal flow of velocity $q_{1}$, with the stationary shock front on the surface. The dif-
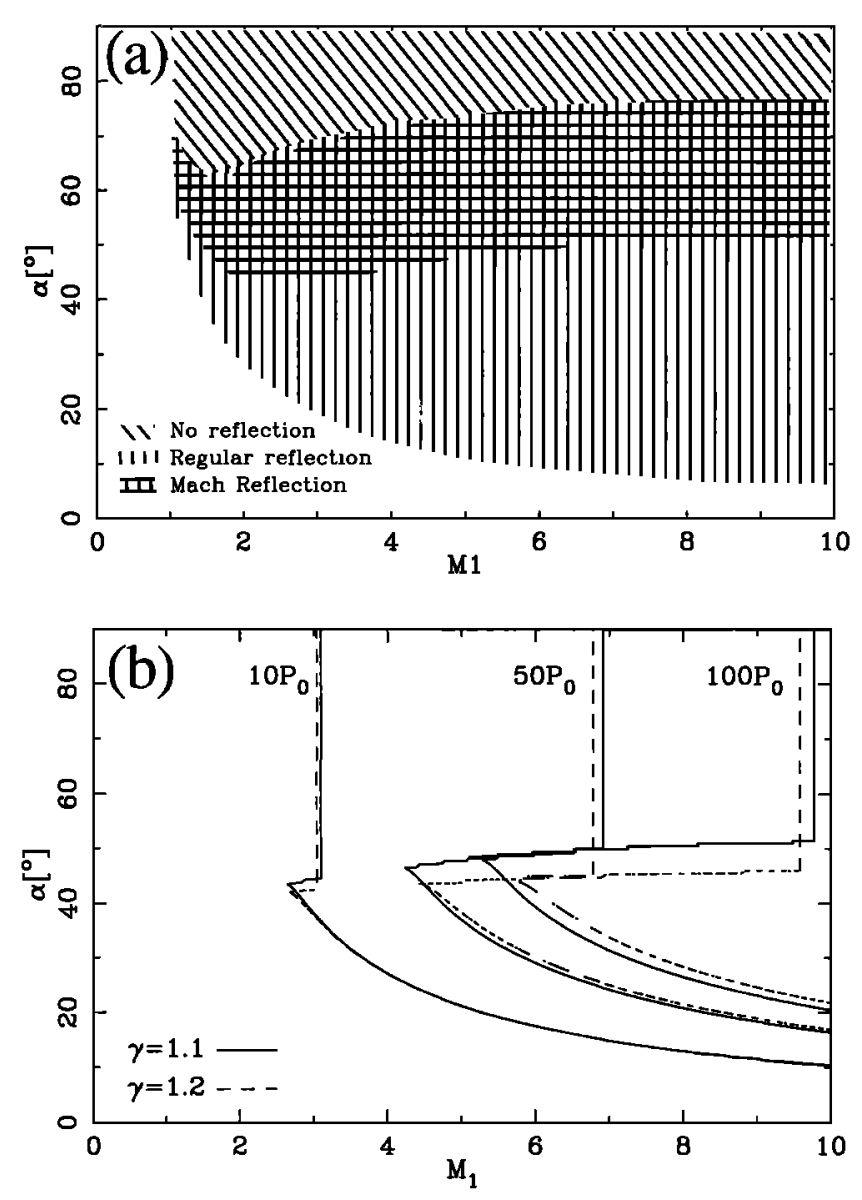

Figure 6. Reflection type and shock pressure as functions of impact angle and impact velocity. (a) Simulated shock configurations for given impact angle $\alpha$ and the propagation velocity $M_{1}$ in the case of $\gamma=1.1$. The various regions represent shock reflection types (b) Comparison of pressure profiles for the cases $\gamma=1.1$ and 12 . 
ference between pseudo-steady flow and a steady flow is that the transition from the regular reflection to the Mach reflection occurs at higher $\alpha$ in the case of the pseudo-steady flow [Hornung, 1986].

Because we approximate the gas flow to be inviscid and the planetary surface to be incompressible, we neglect the shear stress on the surface and the heat flux from (or to) the surface. These effects thicken the viscous boundary layer, and cause the transition from the regular reflection to Mach reflection to occur at higher shock angles of interactions [Hornung, 1986]. This suggests that the boundary effect causes much higher shock pressures and densities in the extended range of the regular reflection. Conversely, the porosity and roughness of the surface attenuate the reflected shock wave. Thus, the calculation of the shocked state can be more complicated on a real planetary surface.

\section{Results}

\subsection{Results of the Paraboloidal Bow Shock Model}

In this section, we will examine shock induced gas properties on the surface caused by the oblique impact of a meteoroid, such as maximum pressure $P(X, Y)$, maximum density $\rho_{s}(X, Y)$, maximum horizontal gas velocity $U(X, Y)$, and maximum temperature $T(X, Y)$, using a paraboloidal bow shock model taking into account reflection shock waves.

Before discussing the effects of the gas shock, we calculate the expected crater radius $R_{c}$ for impacts on Venus. The scaling law of Schmidt [1980] is employed for craters in dry sand which neglects the effect of atmospheric pressure, and the transient radius of the crater, $R_{c t}$, is

$$
\frac{R_{c t}}{R}=0.7\left(\frac{g R}{V_{i}^{2} \sin ^{2} \Phi}\right)^{-016}\left(\frac{4 \pi}{3} \frac{\rho_{m}}{\rho_{t}}\right)^{\frac{1}{3}}
$$

where $g$ is Venus gravity $\left(\simeq 8.8 \mathrm{~m} / \mathrm{s}^{2}\right)$ and $\rho_{m}$ is the density of the meteoroid. We assume a density of the target and the ejecta, $\rho_{t}$, of $2800 \mathrm{~kg} / \mathrm{m}^{3}$. The radius of the final crater, $R_{c}$, becomes $\sim 1.19 R_{c t}$ [Melosh, 1989, p. 129]. Here, obliquity reduces the size of the crater from that of normal impact [Gault and Wedekind, 1978]. Empirically, it is found that the effective impact velocity is approximately equal to the normal component of the impact velocity [R. M. Schmidt, personal communication, 1994].

The resulting shock-induced gas states at the Venusian surface are shown in the case of impact of a stony object at velocity $V_{i}$ of $20 \mathrm{~km} / \mathrm{s}$, with radius $R$ of $1 \mathrm{~km}$, and impact angle of $\Phi=60^{\circ}$ in Figure $7,45^{\circ}$ in Figure $8,30^{\circ}$ in Figure 9 , and $15^{\circ}$ in Figure 10 . The crater radius $R_{c}$ calculated from (13) with $\rho_{m}=2700 \mathrm{~kg} / \mathrm{m}^{3}$ varies from $7.5 R$ to $5.0 R$, as $\Phi$ varies from $90^{\circ}$ to $15^{\circ}$. In these figures, a nominal crater radius, $R_{c} \sim 5 R$, is shown as a dotted circle for reference.

Figures $7 a, 8 a, 9 a$, and $10 a$ demonstrate the shock reflection types corresponding to the bow shock model.
The flow is subsonic in the unhatched region. Since the shock reflection process is nonlinear, the spatial distribution of different reflection modes as a function of impact angle can be observed. The smaller the value of $\Phi$ becomes, the smaller the area of regular reflection, and the greater the surface area which is exposed to a Mach reflection in the up-range area. In the down-range region, or in the case of normal impact, no reflection occurs except at the center of the impact crater because over most of the planetary surface, the shock front intersects the surface with $\alpha \sim 90^{\circ}$. The maximum pressure induced by the shock waves is also shown in Figures $7 \mathrm{a}$, $8 a, 9 a$, and $10 a$. Here, $P_{0}$ is the ambient pressure of the Venusian atmosphere on the surface. There are discontinuities of the isobars in the transition zones between different shock reflection modes in the cases of smaller values of $\Phi$. This corresponds to the discontinuity that can be seen in the transition of Mach reflection from the regular reflection in Figure $6 \mathrm{~b}$. The pressure produced by atmospheric shock is $\sim 10^{2} \mathrm{GPa}$ in the center of a crater and $\sim 10^{1} \mathrm{GPa}$ at the rim of a crater for the impact of a $1-\mathrm{km}$-radius meteoroid at $20 \mathrm{~km} / \mathrm{s}$ impact velocity.

The maximum gas density compressed by the shock, $\rho_{s}(X, Y)$, is shown in Figures $7 \mathrm{~b}, 8 \mathrm{~b}, 9 \mathrm{~b}$, and $10 \mathrm{~b}$. There are discontinuities of the profiles in the transition zones between different shock reflection modes in the cases of smaller $\Phi$, as can also be seen in the pressure profiles. The lower the impact angle $\Phi$ becomes, the wider the region of high density in the up-range direction.

The maximum horizontal gas particle velocity generated by the shock wave $U(X, Y)$ is plotted in Figure $7 c, 8 c, 9 c$, and $10 \mathrm{c}$ for different $\Phi$. A considerable area (e.g., within $\sim 20 R_{c}$ for $\Phi=30^{\circ}$ ) is affected by the strong $(>100 \mathrm{~m} / \mathrm{s})$ gas velocity in this case. The direction of the gas flow is also shown in Figure $7 \mathrm{c}, 8 \mathrm{c}$, $9 \mathrm{c}$, and $10 \mathrm{c}$ with small ticks. Initially strong gas flow occurs radially from the center of the impact. In the case of oblique impacts, this flow changes to become perpendicular to the incoming trajectory of the body in the up-range region.

Figures 7d, 8d, 9d, and 10d show the maximum dynamic pressure, $\rho_{s} U^{2}$, calculated from the results of Figures $7 b, 8 b, 9 b$, and $10 b$ and Figure $7 c, 8 c, 9 c$, and $10 \mathrm{c}$. The maximum dynamic pressure is $\sim 10^{2} \mathrm{GPa}$ at the center of the impact. Since the ranges of the dynamic pressure are affected by the gas density and the gas particle velocity, as $\Phi$ or the radial distance from the center decreases, the contours change from circular to shapes which are constricted around the center of the impact along the up-range footprint of the projectile trajectory. This bilateral symmetry was previously qualitatively estimated by Ivanov et al. [1986].

Horizontal gas flow induces viscous stresses, which in turn, produce surface disturbance. Figures of gas particle velocity and dynamic pressure indicate that, in the case of larger values of $\Phi$, the materials can be transported radially on the planetary surface around the crater. In the case of smaller $\Phi$, in the up-range 

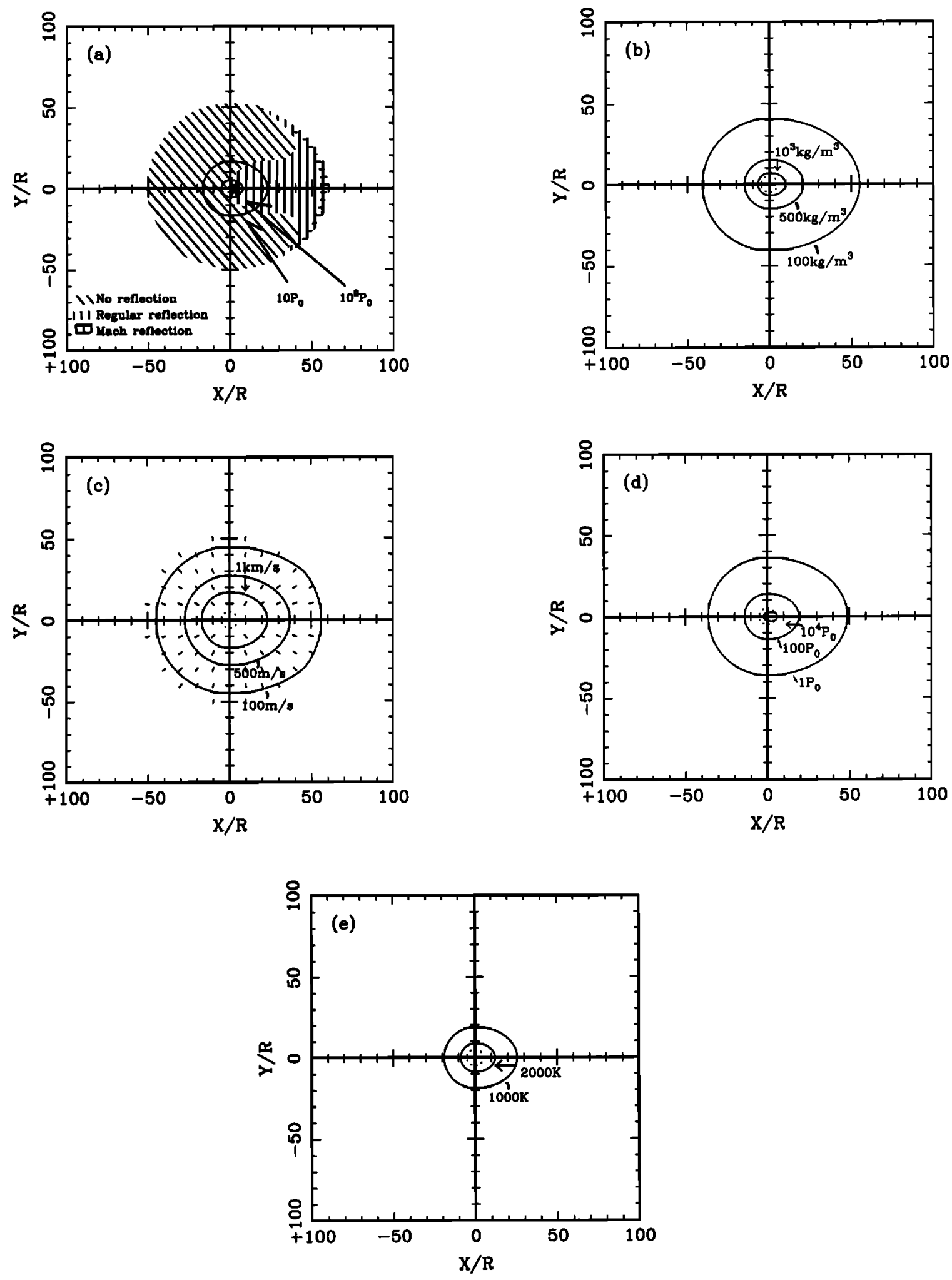

Figure 7. Surface maps of shock properties for oblique impact on Venus in the case of the impact velocity of meteoroid $V_{i}=20 \mathrm{~km} / \mathrm{s}$. The impact angle is $\Phi=60^{\circ}$. Coordinate system is the same as that of Figure 4. The dotted circles represent the crater size in the case of impact by a $1 \mathrm{~km}$ radius meteoroid. (a) Shock reflection types and maximum shock pressure. The representation of reflection types is the same as Figure 6. Isobars are normalized by Venusian surface pressure $P_{0}$ of $9.2 \mathrm{MPa}$. (b) Maximum shock density. (c) Maximum horizontal gas velocity induced by shock wave. Small ticks in the figure show the unit vectors of gas flow directions. (d) Maximum induced dynamic pressure with the unit of $P_{0}$. (e) Maximum shock-induced gas temperature. $T_{0}$ is Venusian surface temperature, $735 \mathrm{~K}$. 

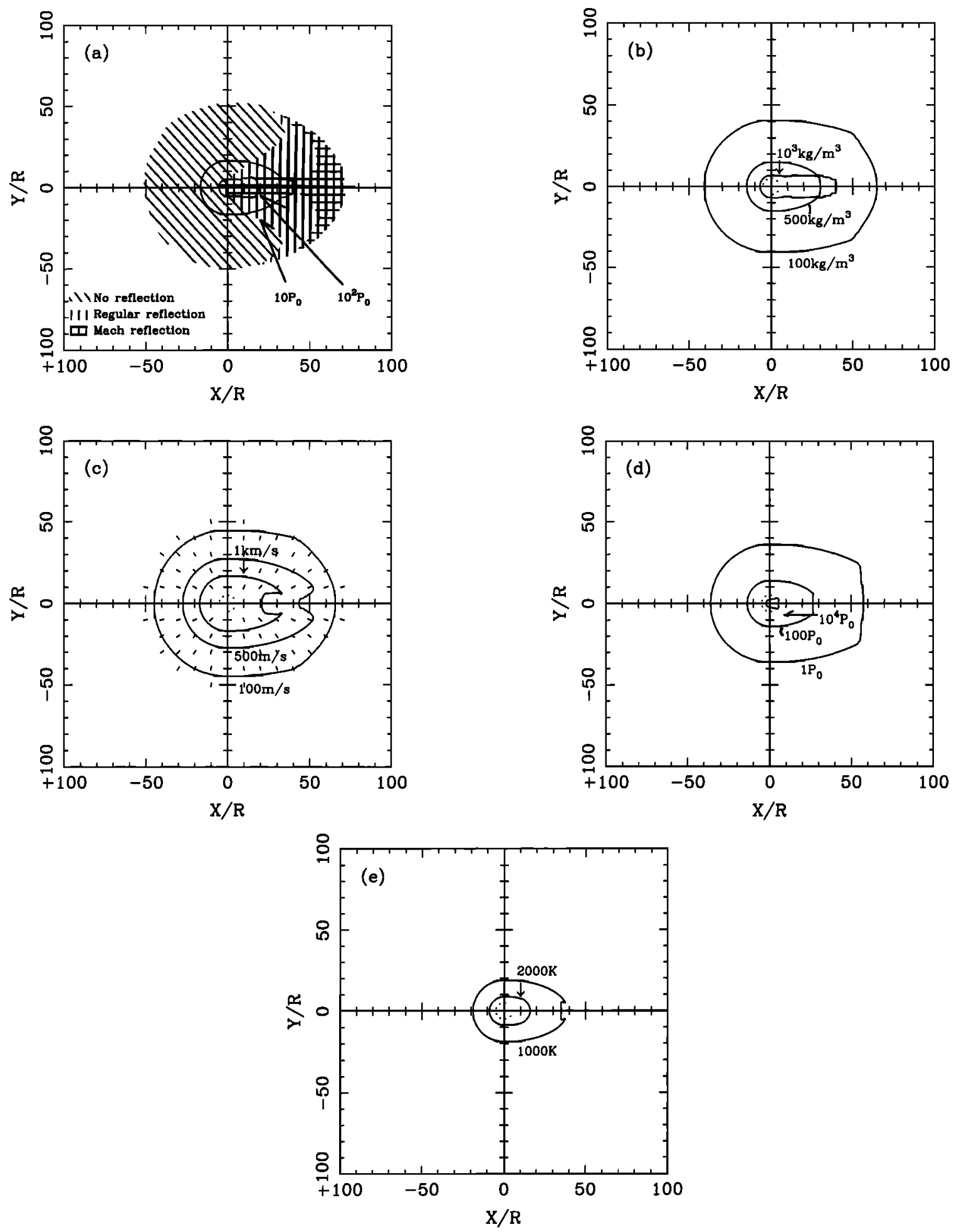

Figure 8. Same as Figure 7 , but for $\Phi=45^{\circ}$.

region, the gas flow moves materials toward the perpendicular to the trajectory footprint rather than dispersing them radially from the center of the impact. This can cause the material to be transported toward the incoming direction and in the direction which is perpendicular to the trajectory footprint. Thus particle motion can cause elongation and bilateral distributions of surface material.
Although there may be significant atmospheric effects of blast waves on the surface of Venus, the thermal energy content of these gas shocks is insufficient for production of significant thermal metamorphism outside the range of the crater ejecta. For example, in the case of $V_{2}=20 \mathrm{~km} / \mathrm{s}$ and $R=1-\mathrm{km}$, the gas temperatures are less than $\sim 2000 \mathrm{~K}$ over areas not covered by crater ejecta (Figures 7e, 8e, 9e, and 10e). 

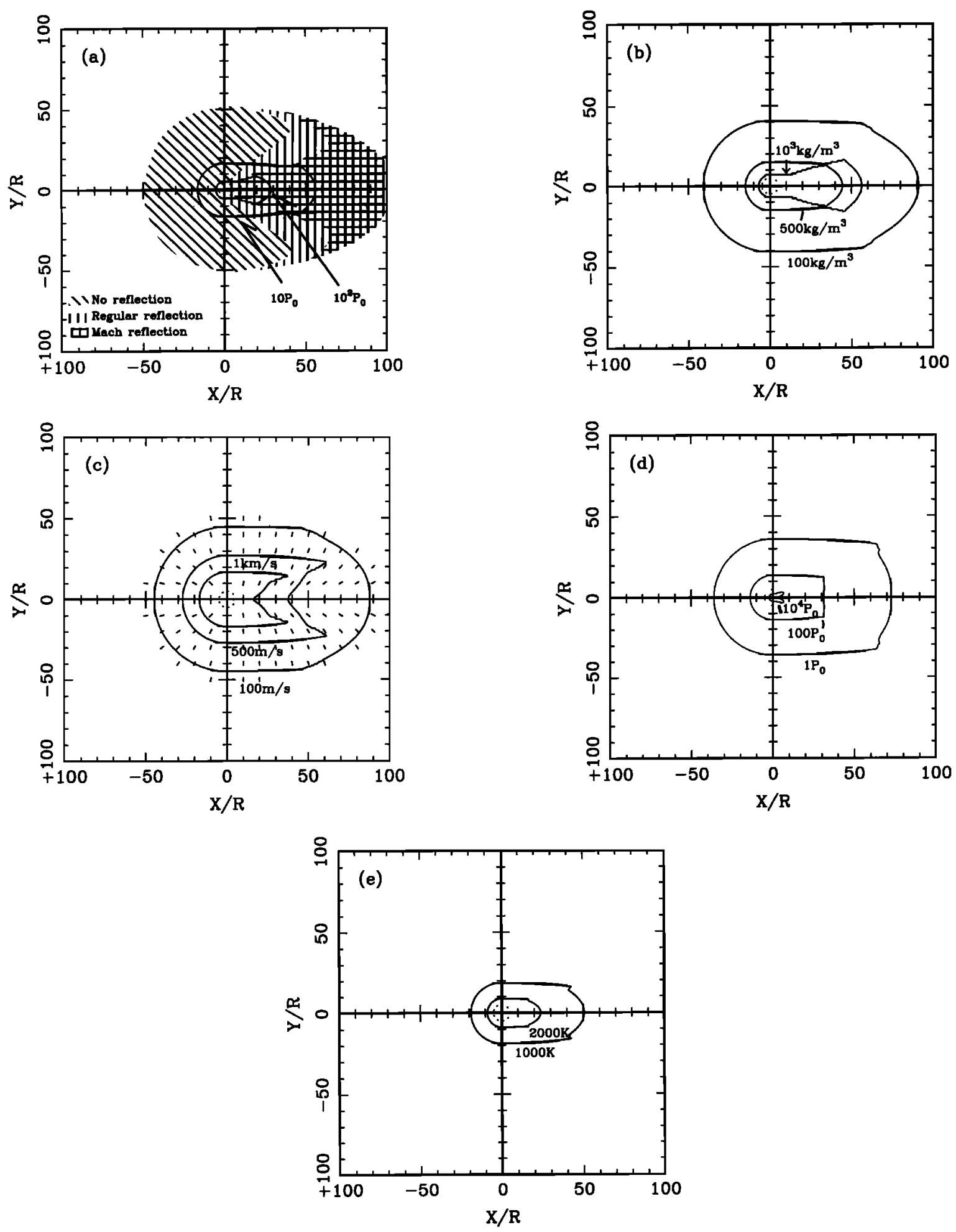

Figure 9. Same as Figure 7, but for $\Phi=30^{\circ}$.

From Figures 7-10, it is clear that the region affected by the shock wave becomes wider and more elongated in the up-range region with decreasing $\Phi$, that is, with greater obliquity of impact.

The effects of the impact velocity on the shock properties along the footprint of the impactor's trajectory in the up-range direction $(X>0$ and $Y=0$ in (5)) are shown for the case of $\Phi=45^{\circ}$ in Figure 11 .

Figure 11a shows the profiles of the maximum pressure. There is a kink in the pressure profile of $V_{i}=50$ $\mathrm{km} / \mathrm{s}$ in Figure 11a because of the transition from the regular reflection to Mach reflection. The maximum rel- 

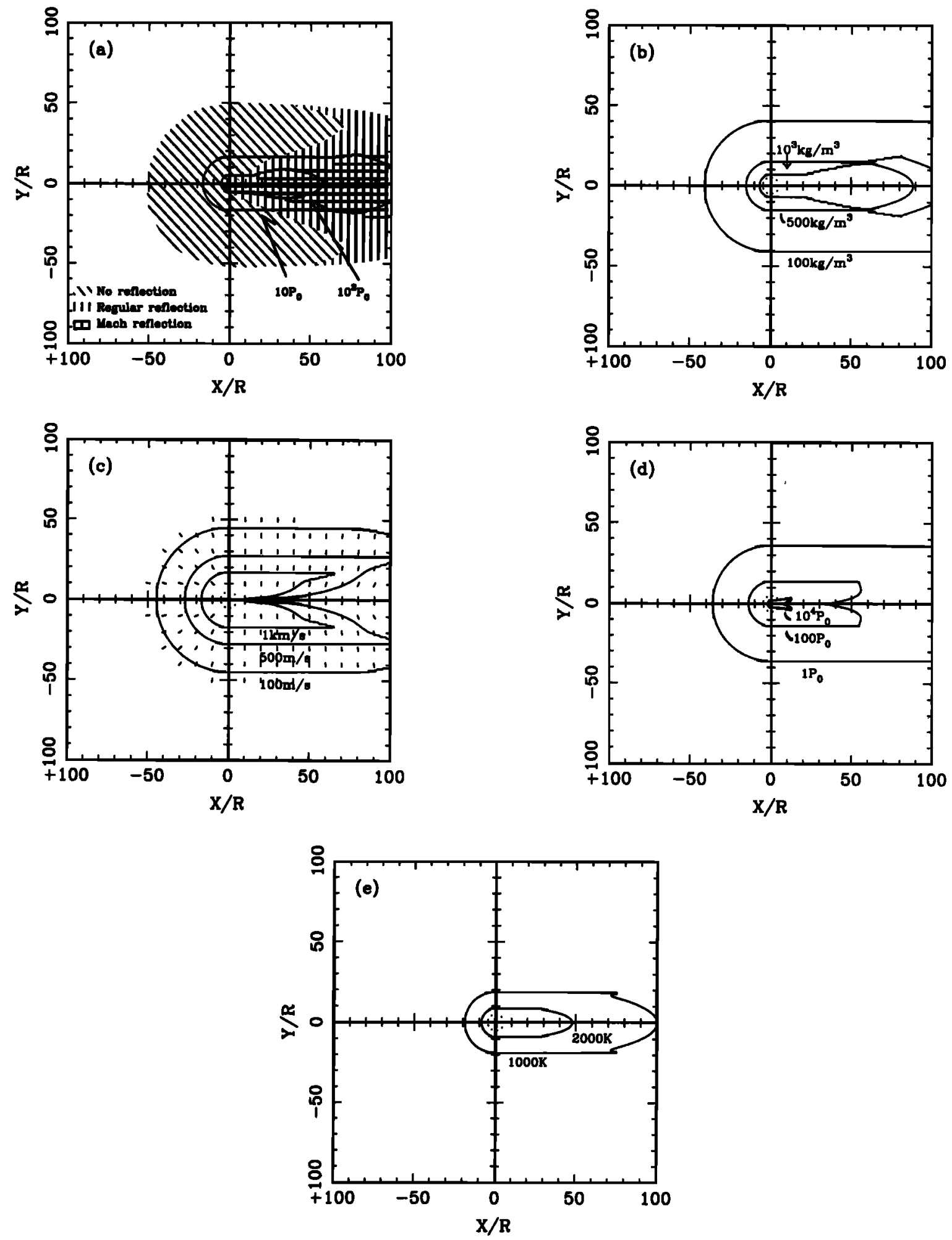

Figure 10. Same as Figure 7, but for $\Phi=15^{\circ}$.

ative compressed density is shown in Figure 11b. Here $\rho_{0}=65 \mathrm{~kg} / \mathrm{m}^{3}$. Figure $11 \mathrm{c}$ shows the maximum horizontal gas particle velocity for various impact velocities. The gas velocity decreases more rapidly at the region close to the subsonic region. The horizontal gas velocity becomes zero, and the corresponding kink exists on the tangential contact point of the bow shock to the surface (e.g., at $X / R \sim 1.4$, in the case of $\Phi=45^{\circ}$ ), which is nearly inside the crater. The dynamic pressure is normalized by $P_{0}$ in the case of Figure 11d. In Figure $11 \mathrm{e}$, maximum relative shock-induced gas temperature is normlized to Venusian surface temperature, $T_{0}=735$ $\mathrm{K}$.

For constant ratios of target and impactor densities and for a given value of $\Phi$, the ratio of the radius of the crater to that of the impactor is proportional to 

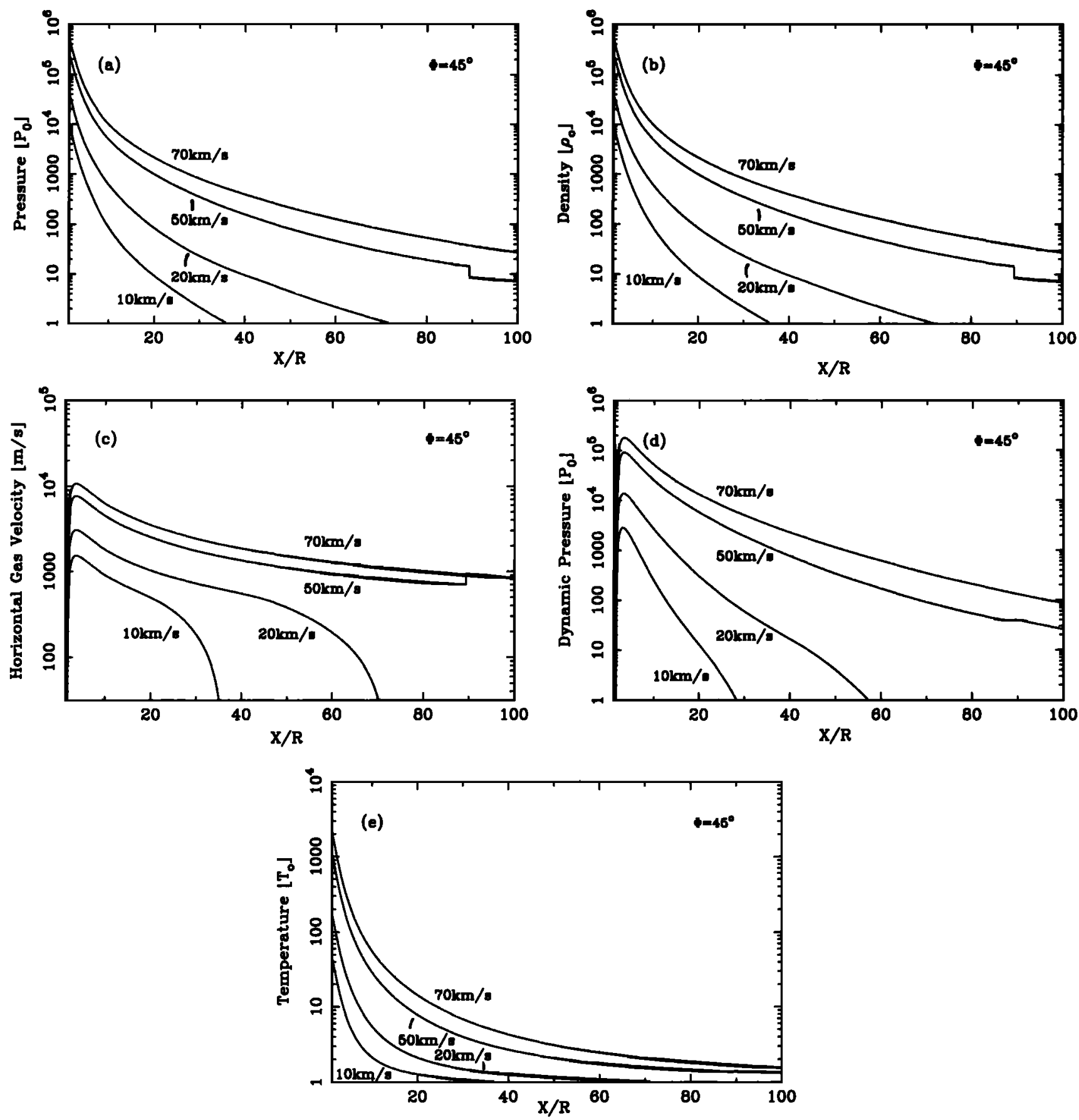

Figure 11. Shock properties for various impact velocities in the case of impact angle $\Phi$ as a function of distance from the center of the crater toward the incoming direction, along the trajectory footprint. Impact velocities $V_{i}$ of $10 \mathrm{~km} / \mathrm{s}, 20 \mathrm{~km} / \mathrm{s}, 50 \mathrm{~km} / \mathrm{s}$, and $70 \mathrm{~km} / \mathrm{s}$ are selected. (a) Maximum shock pressure normalized by $P_{0}$. (b) Maximum shock density normalized by the initial gas density of $\rho_{0}$. (c) Maximum horizontal gas velocity. (d) Maximum dynamic pressure normalized by $P_{0}$. (e) Maximum gas temperature normlized by the initial gas temperature of $\mathrm{T}_{0}$.

$\sim V_{i}^{032}$ from (13). In the case of normal impact, or the case without modification by the mode of reflection, the distance of the subsonic region (e.g., $r / R$ for $M_{1} \sim 1$ ) varies as $\sim V_{i}$. Therefore the region affected by the gas shock compared to the radius of the crater becomes wider, as the impact velocity increases.

\subsection{Gas Properties of the Atmosphere}

We have used the effective ratio of the specific heat $\gamma$ instead of the exact ratio of the specific heat for a perfect gas, and used the value of $\gamma=1.1$ for $\mathrm{CO}_{2}$ in our calculations. In the case of triatomic $\mathrm{CO}_{2}$ gas, $\gamma$ is 
1.20 at $730 \mathrm{~K}$ and 1.15 at $2900 \mathrm{~K}$ at 1 atm [Hilsenrath et al., 1955], and the variation of $\gamma$ depends primarily on the temperature. However, the density behind the bow shock can reach higher values in the case of an ideal dissociating gas than in the case of a perfect gas if significant amounts of dissociation occur behind the shock and $\gamma$ decreases close to unity [Vincenti and Kruger, 1965, p. 182]. In the case of $\mathrm{CO}_{2}$, the calculation of the ideal dissociating gas for $M \sim 12$ shows that the ratio of the density before and behind the shock becomes $\sim 23$ in the state of the dissociative relaxation, and the corresponding $\gamma$ becomes 1.09 (H. Hornung, unpublished data, 1992). Therefore, in order to take into account real gas effects, we used a smaller effective ratio, $\gamma$, rather than the value expected for the perfect gas. From Figure $6 \mathrm{~b}$, in the case of smaller $M(<5)$, the effect of the different $\gamma$ to the shock properties is not significant, but for large $M$, the effect of dissociation is important. The differences in shock properties in the case of a different value of $\gamma$ to those of $\gamma_{0}=1.1$ are roughly $P(\gamma) / P\left(\gamma_{0}\right)=1.9 \gamma /(\gamma+1)$ for shock pressure and $\rho(\gamma) / \rho\left(\gamma_{0}\right)=21(\gamma-1) /(\gamma+1)$ for shock density for normal impact. Therefore the pressure does not change significantly. However, the dynamic pressure changes about a factor of 2 to 4 . If the gain of the enthalpy increases further to dissociate the gas completely, the gas becomes monatomic; moreover, electronic excitation becomes significant at $T>10^{4} \mathrm{~K}$. Then, $\gamma$ increases up to the value of $5 / 3$. In the region immediately outside of a crater within the ejecta blanket, the gas temperature is not high enough to dissociate the gas completely (e.g., Figures $7 \mathrm{e}, 8 \mathrm{e}, 9 \mathrm{e}, 10 \mathrm{e}$, and $11 \mathrm{e}$ ), and the constant $\gamma \sim 1.1$ is a reasonable value to utilize for describing the shock-induced gas over a wide area.

\subsection{Time Dependence of Shock Properties}

We have estimated the maximum shock properties at each point of the planetary surface; however, in the case of a blast wave expansion, the shock properties at a given position are functions of time. The pressure increases suddenly to our calculated peak value upon shock arrival. Attenuation then occurs, and the shock pressure decreases below the ambient value upon the arrival of the rarefaction wave. The pressure, then, relaxes to the ambient state. Attenuation is estimated using the axisymmetric power laws shown in Figure $2 \mathrm{~b}$, due to the similarity of the bow shock model to the axisymmetric blast wave. In the following discussion, we estimate the time variable gas shock properties by applying the method of Zel'dovich and Raizer [1966] to the case of a cylindrical blast wave.

The distance of the shock wave from the center of the impact normalized by $R$, at time $t$ after the impact, $r_{s}(t)$, and its propagation velocity, $d r_{s} / d t(t)$, is, from (8), proportional to $t^{1 / 2}$ and $t^{-1 / 2}$, respectively. Assuming that the shock wave arrives at $t_{0}(<t)$ and $t$ at a distance of $r_{s}\left(t_{0}\right)$ and $r_{s}(t)$, the ratio of the maximum pressure, density, and gas particle velocity at time $t$ to those at $t_{0}$ are: $P_{\max }(t) / P_{\max }\left(t_{0}\right) \sim$ $t_{0} / t, \rho_{\max }(t) / \rho_{\max }\left(t_{0}\right) \sim 1$, and $U_{\max }(t) / U_{\max }\left(t_{0}\right) \sim$ $\left(t_{0} / t\right)^{1 / 2}$. Here, the subscript "max" indicates the maximum value along the radial direction at each time or at the shock front. The ratios of the attenuated shock properties at $r_{s}\left(t_{0}\right)$, at time $\mathrm{t}$ relative to the maximum shock properties at $r_{s}(t)$, is expressed by the axisymmetric power law; $P(t) / P_{\max }(t) \sim \eta\left(y_{0}\right)$, $\rho(t) / \rho_{\max }(t) \sim \zeta\left(y_{0}\right)$, and $U(t) / U_{\max }(t) \sim \xi\left(y_{0}\right)$, as seen in Figure $2 \mathrm{~b}$. Here, $\eta, \zeta$, and $\xi$, are the similarity functions calculated in Figure $2 b$, for the pressure, density, and velocity and $y_{0}=r / r_{s}$.

Therefore, at the position of $r_{s}\left(t_{0}\right)$, the shock properties as a function of time are given approximately as

$$
\begin{aligned}
P(t) & \sim P_{\max }\left(t_{0}\right)\left(t_{0} / t\right) \eta\left(\left(t_{0} / t\right)^{1 / 2}\right) \\
\rho(t) & \sim \rho_{\max }\left(t_{0}\right) \zeta\left(\left(t_{0} / t\right)^{1 / 2}\right) \\
\text { and } & \\
U(t) & \sim U_{\max }\left(t_{0}\right)\left(t_{0} / t\right)^{1 / 2} \xi\left(\left(t_{0} / t\right)^{1 / 2}\right)
\end{aligned}
$$

when $t \geq t_{0}$. At $t<t_{0}, P$ and $\rho$ are equal to the initial ambient values, and $U=0$. Figure $2 \mathrm{~b}$ shows that the function of $\eta$ becomes a constant $\sim 0.47$, as $y_{0}<0.8$, and $\xi\left(y_{0}\right) \sim y_{0}$, so we can approximate the pressure and the velocity as $P(t) \sim 0.5 P_{\max }\left(t_{0}\right) t_{0} / t$ and $U(t) \sim U_{\max }\left(t_{0}\right) t_{0} / t$ at the same position. For example, in the case of a $1-\mathrm{km}$ meteoroid impacting at $20 \mathrm{~km} / \mathrm{s}$, at a radial distance of $\sim 5 R_{c}\left(R_{c}\right.$ is crater radius), some 20 $s$ are required to reduce the horizontal particle velocity on the planetary surface to one-half of its initial value. Although the shock wave is a transient phenomenon, its attenuation becomes gradual at large distances from the center of the crater. Thus at larger distances from the center of the crater, if the gas shock achieves a sufficient magnitude to cause the disturbance of the surface properties, the time duration of near peak shock properties of the same magnitude occurs over time scales comparable to the time required for the shock to propagate over the radial distance along the surface which is disturbed. We therefore infer that the maximum shock properties examined in present work provide first approximations to shock disturbances on the Venusian surface.

\subsection{Entrainment of Surface Materials}

In this section the magnitude of the surface disturbance is estimated using the shock properties obtained in section 3.1. The change of the surface features around the site of large near-surface explosions is mainly caused by deposition of thrown-out ejecta and by deposition of the debris (dusts, pebbles, rocks, and boulders) lofted outside the crater by the high-speed horizontal gas flow associated with the blast wave. The viscous stresses associated with this gas flow above the surface entrain and loft the surface materials, and resulted in the turbulent mixing of dust and pebbles with the atmosphere. Then surface materials are scoured and are redeposited. This process changes the original surface features. We assume that the effects on a planetary surface induced by impact cratering are analogous to the effects of large near-surface explosions. The present bow shock model demonstrates that a wide region, compared to the crater radius, can be affected by the shock wave and the fol- 
lowing horizontal high-velocity gas flow. Although this high-speed gas flow is transient, it is expected to loft surface materials and change the surface properties.

In order to examine how wide a surface area can be affected by the impact, we will employ the shock propertıes of the gas calculated from the bow shock model to the saltation or suspension regime.

The horizontal gas particle velocity $U$ is related to the wind friction velocity $u_{*}$ by a friction coefficient $C_{f}^{\prime}$. For the case of a Reynolds number, $R e \simeq 10$, for steady flow, $u_{*}$ is

$$
u_{*}^{2}=\frac{1}{2} C_{f}^{\prime} U^{2}
$$

[Iversen et al., 1976].

We note that $C_{f}^{\prime \prime}$ changes from $\sim 10^{-4}$ to $\sim 10^{-2}$ depending on the roughness of the surface and the type of flow (steady versus transient) [Rosenblatt, 1985]. We use $C_{f}^{\prime \prime} \sim 0.0015$ for turbulent flows with $R e \sim 10^{9}$ $\left[\right.$ Tant, 1967] for the case in which the value of $\mathrm{CO}_{2}$ viscosity is $\sim 10^{-5}$ Pas [Whate, 1974] and $U \sim 10^{3} \mathrm{~m} / \mathrm{s}$, $\rho \sim 10^{2} \mathrm{~kg} / \mathrm{m}^{3}$ and rock size $D_{p} \simeq 10^{-1} \mathrm{~m}$. $C_{f}^{\prime}$ becomes 0.003 for $\operatorname{Re}=10^{7}$; therefore the value we use is a lower limit.

The threshold friction velocity, $u_{* t}$, that is the friction velocity required to initiate lofting and saltation of a particle of diameter, $D_{p}$, can be written as

$$
u_{* t}=A\left[\frac{\left(\rho_{p}-\rho\right)}{\rho} g D_{p}\right]^{\frac{1}{2}}
$$

where $\rho_{p}$ is the particle density $\left(\simeq 2800 \mathrm{~kg} / \mathrm{m}^{3}\right), \rho$ is gas density, and the coefficient $A$ is estimated as 0.11 for 1- $\mu \mathrm{m}$ - to 1-cm-diameter particles [Iversen et al., 1976]. We assume that the value of the coefficient is effective for $10^{0}-10^{1} \mathrm{~cm}$ sized rocks, although no experimental work for rocks with diameters greater than $1 \mathrm{~cm}$ has been conducted. We apply this equation to the gas flow induced by the paraboloidal bow shock wave. By setting $u_{*}=u_{* t}$ in (15) and (16), we can estimate the maximum saltation rock size with given gas velocity. This can be applied to the region where the propagation of the shock wave becomes supersonic, as the horizontal gas particle velocity is also comparable and it decreases less rapidly.

In Figure 12, maximum saltation rock diameter is calculated for $V_{\imath}=20 \mathrm{~km} / \mathrm{s}$ and $\Phi=30^{\circ}$ using the maximum gas velocity, density profiles, and dynamic pressure of Figure 9. In this case, the dynamic pressure of $1 \mathrm{MPa}$ can induce saltation of boulders up to the order of $1 \mathrm{~m}$ in size. Moreover, rocks of le ss than $10 \mathrm{~cm}$ in size can be saltated within most of the area of the supersonic region. Particles of several millimeters in the maximum size can be moved easily by Venusian surface winds [Greeley et al., 1984]. Therefore, surface winds cannot explain the lofting of particles of more than 1 $\mathrm{cm}$ in size; however, impact processes can account for the movement of such large boulders.

In the case of steady flow, the transport distance of the particles is $\sim 10 u_{*}^{2} / g$, if the final velocity, $w_{F}$, is

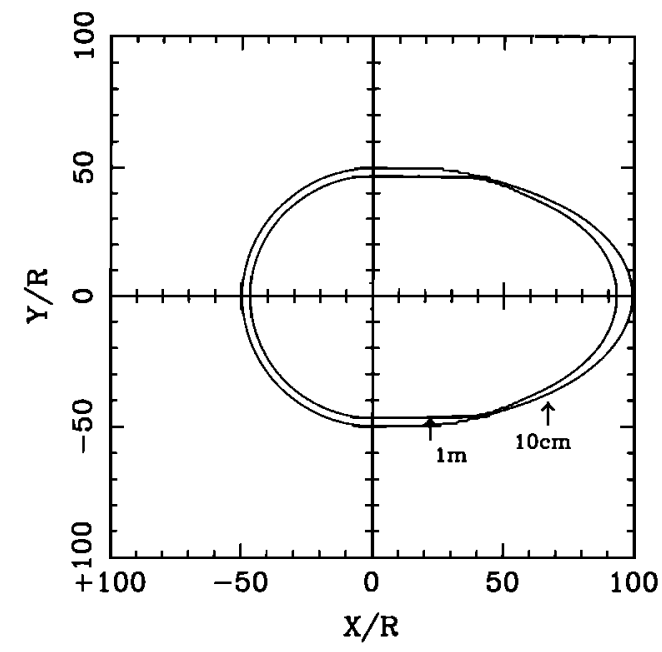

Figure 12. Maximum saltation boulder size calculated in the case of impact velocity $V_{i}=20 \mathrm{~km} / \mathrm{s}$ and impact angle $\Phi=30^{\circ}$ using profiles in Figure 9 . The crater size estimated from equation (13), $R_{c}=5 R$, is shown as a dotted line.

less than $u_{*}$ [Bagnold, 1941]. The transport distance is expected to be less than this estimate because of the transient flow of the shock. In general, the final velocity decreases with the size of the particle.

\subsection{Degree of Mutual Comminution}

Particles of surface materials, placed in motion by the radial flow produced by the atmospheric shock, can be comminuted via mutual collision and collisions with the planetary surface resulting in a fine-grained porous deposit. In this section, we will estimate the degree of mutual comminution of the entrained materials in the gas flow produced by the bow shock analogous to impact fragmentation.

The diameter of the largest fragment, $D_{p L}$, produced by the collision of two objects composed of the same materials with diameters of $D_{p p}$ and $D_{p t}$ was found to be [Mrzutani et al., 1990]

$$
\left(\frac{D_{p L}}{D_{p t}}\right)^{3}=0.024\left(\frac{P_{i} D_{p p}^{3}}{Y D_{p t}^{3}}\right)^{-093}
$$

This is applicable if $D_{p p} \leq D_{p t}$, where $Y$ is an effective fracture strength of the rock and $P_{2}$ is the impact pressure calculated from the Hugoniot equation of $P_{z}=\rho_{p} u_{p} c_{l}$ in the case of $u_{p} / c_{l} \ll 1$. Here $c_{l}$ is the longitudinal elastic velocity of the particle material and $u_{p}$ is the relative velocity of the particles. This shock pressure is also applicable to the case of low impact velocity of $\leq 10 \mathrm{~m} / \mathrm{s}$ within a factor of 2 . We use the values for basalt in Mrzutanı et al. [1990], which are $Y$ $=480 \mathrm{MPa}$ and $c_{l}=5040 \mathrm{~m} / \mathrm{s}$.

The rock particles, once entrained, continuously collide with each other until landing on the planetary surface. They can also impact obliquely. However, we assume that every ejecta particle collides normally, at 
least once, with a particle of the same or larger size with a characteristic velocity. We considered two characteristic relative velocities. One occurs when the entrained material falls onto the Venus surface and impacts rocks on the surface. Another occurs when the surface materials are entrained and impact other rocks in the flow.

In the first case, the impact velocity is almost the same order as the final velocity, $w_{F}$, which is calculated as

$$
3 C_{D} \rho w_{F}^{2}=4 D_{p}\left(\rho_{p}-\rho\right) g
$$

where $C_{D}$ is 0.5 in the case of $w_{F}<10^{1} \mathrm{~m} / \mathrm{s}$ and $R e \sim 10^{4}$ [White, 1974]. Then the ratio of the largest diameter of the post-collisional fragment to the initial diameter, $D_{p L} / D_{p}$, becomes $\sim 0.4$ when $D_{p} \sim 10 \mathrm{~cm}$, and $D_{p L} / D_{p}$ becomes $\sim 0.6$ when $D_{p} \sim 1 \mathrm{~cm}$.

In the latter case, the impact velocity is almost equal to the initial upward saltation velocity $w$. Since $w$ is the same order as $u_{*}$ [Bagnold, 1988], we assume collision velocity $u_{p} \sim u_{*}$. In the case of impact velocity of $20 \mathrm{~km} / \mathrm{s}$ and impact angle of $30^{\circ}$ as in Figure 12, the calculated ratio of the largest fragment diameter to the initial diameter, $D_{p L} / D_{p}$, for $D_{p}=10 \mathrm{~cm}$ is shown in Figure 13. The degree of mutual collision is effective in the region close to the crater (the particle diameter is reduced by $50 \%$ at $\sim 40 \mathrm{R}$ and reduced to $25 \%$ at $\sim 15 \mathrm{R}$ ) and in the region of the hypersonic flow propagation. However, mutual collisions are less effective in outer parts of the supersonic flow regions and are ineffective in subsonic regions.

Both cases may occur wherever the diameters of surface materials allow lofting and saltation or suspension. The surface features are thus changed by the shockinduced gas flow. This calculation shows that highimpact velocity produces a wider area affected by saltation, crushing and redistribution of surface materials due to lofting, turn-over, and the mutual collision of

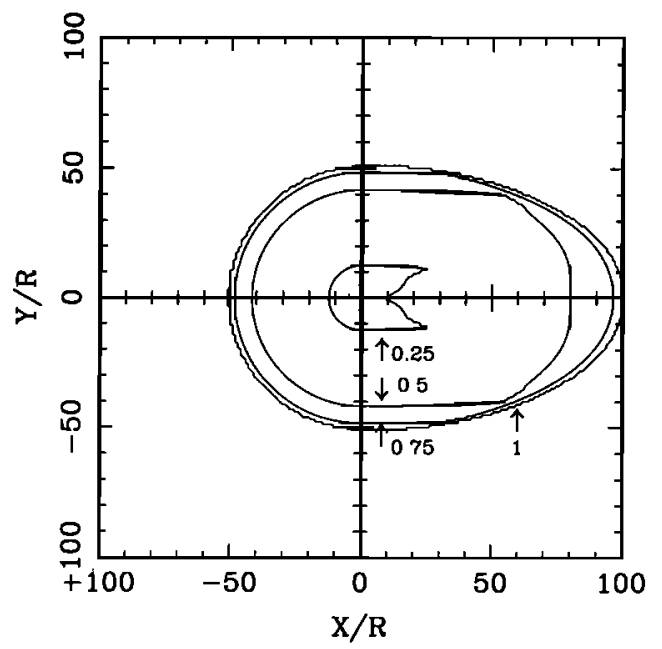

Figure 13. Contours of the ratio of the largest diameter of the post-collisional surface particles relative to the diameter of original target particles, $D_{p L} / D_{p t}$, in the case of collision with particles of the same size. Impact parameters are the same as in Figure 12. rocks, and that oblique impact induces a more elongated affected area, which is oriented toward the uprange direction. This is consistent with the observation of halos that elongate toward the opposite direction of the ejecta distribution, as shown in Figure 1.

\subsection{Wide Crater Halos}

From the analysis of backscatter cross sections of crater halos presented in section 1, the presence of dark halos around craters implies that the surface is $\sim 30 \%$ smoother than the original surface. Bright halos, in contrast, indicates a $\sim 30 \%$ rougher surface than initially present.

Applying the effect of atmospheric shock investigated in the previous section to the feature of dark halos surrounded by the bright halo, we infer that the dark halo represents finer ground regolith produced by comminution via mutual collision. The resulting smoother porous surface results in a lower radar backscatter cross section than the original surface in the vicinity of the crater. In contrast, bright halos are caused by an increase in roughness of the surface materials. At the greater radii where the radar-bright halo occurs, the saltation is not as effective as producing fine materials by fracture of boulders on the surface. Instead boulders may be turned over and redeposited, whereas the small amount of finer particles are deposited between large boulders or diffused over a wider area. Thus a roughened surface can be induced by the blast wave which will result in a higher radar backscatter cross section than that of the original surface (Figure 14).

\section{Discussion}

\subsection{Application to the Venusian Craters}

In this section, we apply the results of the bow shock model to the craters shown in Figure 1 in order to test the radar dark/bright halos models described in the previous section.

The ratio of the distance from the crater's center to the boundary of the supersonic flow region, $r_{t}$, to the meteoroid's radius, $R$, becomes a linear function of the impact velocity in the case of the vertical impact from (5a) and (6), as

$$
\frac{r_{t}}{R} \simeq 2.5 V_{i}
$$

Here, $V_{\imath}$ is in units of $\mathrm{km} / \mathrm{s}$. Using the scaling law of (13), the radius of the crater $R_{c}$ becomes $\sim 5 R$ for $R=1$ $\mathrm{km}, \rho_{m}=10^{3} \mathrm{~kg} / \mathrm{m}^{3}$, and $V_{i}=20 \mathrm{~km} / \mathrm{s}$; the ratio of the radius of the supersonic shock region to the radius of the crater $r_{t} / R_{c}$, then becomes $\sim 10-17$ with $15^{\circ}<$ $\Phi<60^{\circ}$. The ratio of the shortest distance from the center of the crater to the boundary $r_{t s}$ to the longest distance $r_{t l} r_{t s} / r_{t l}$, becomes approximately $\sin \Phi$ from (6), if $M_{1} \gg 1$.

We assume that the initial Venusian surface contains a range of grain sizes less than the order of $10 \mathrm{~cm}$. In the region where particles of sufficient diameter (a 

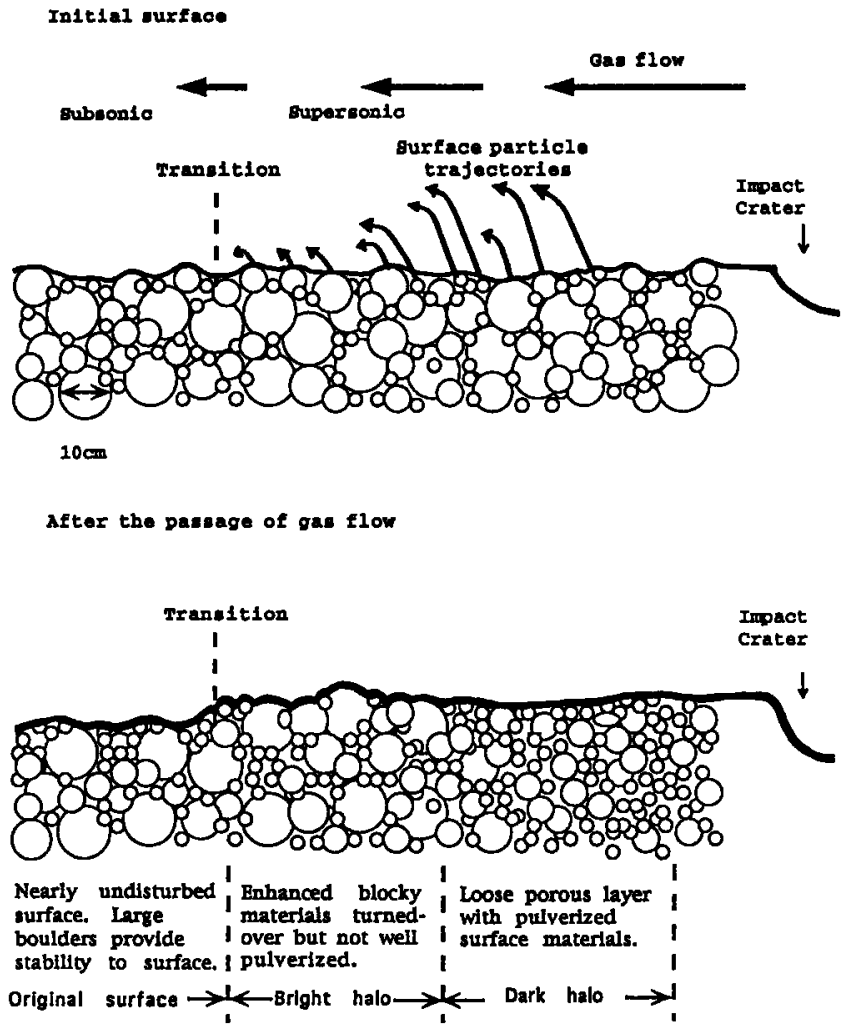

Figure 14. Sketch of the surface disturbances by the impact induced gas flow as a function of the distance from the crater center. We assume that the surface consists of particles of less than $10 \mathrm{~cm}$ in size.

few centimeters to $\sim 10 \mathrm{~cm}$ ) cannot be entrained and suspended, finer particles will be deposited upon and between the large cobbles; then they can be lofted. Thus the effect of the disturbance of the surface by the gas shock is expected to be small, maintaining approximately the original radar properties (Figure 14). Therefore we assume that the transition from the undisturbed area to the disturbed area in radar properties occurs approximately in the locations where the maximum saltation boulder diameter is about $10 \mathrm{~cm}$, and this is approximately the boundary between the supersonic flow region and subsonic flow region (Figures $9 \mathrm{a}$ and 12).

Then the (19) becomes

$$
R_{c} / R=2.5 V_{i}\left(R_{c} / r_{h s}\right)
$$

where $r_{h s}$ is the shortest radial size of the halo and is defined in Figure 15 along with the longest radial distance $r_{h l}$. We can observe the radii of the halos, $r_{h}\left(r_{h s}, r_{h l}\right)$ and $R_{c}$. Therefore (20) can provide the relationship between the impact velocity and the radius of the meteoroid, and the observed radius of the halo around the crater.

In the case of the crater in Figure 1a, the longest radial distance of the bright halo, $\mathrm{r}_{h l}$, and the shortest radial distance, $\mathrm{r}_{h s}$, are approximately $74 \mathrm{~km}$ and 50 $\mathrm{km}$, respectively. Their ratio corresponds to an impact angle $\Phi$ of $\sim 43^{\circ}$ (Figure 15). The radius of the crater $R_{c}$ is $\sim 3100 \mathrm{~m}$. If we assume that the crater was in- duced by an icy comet of a density of $1000 \mathrm{~kg} / \mathrm{m}^{3}$, the feature corresponds to an impact velocity on the surface $V_{i}$ of $\sim 45 \mathrm{~km} / \mathrm{s}$ and $R$ of $\sim 450 \mathrm{~m}$. The corresponding incident velocity of $\sim 55 \mathrm{~km} / \mathrm{s}$ is obtained by applying the classical ablation model of Bronshten [1983]. The solar revolution velocity of Venus and that of typical short-period comets at Venusian orbit are $35 \mathrm{~km} / \mathrm{s}$ and $\sim 45 \mathrm{~km} / \mathrm{s}$, respectively. Therefore the impact velocity of $\sim 55 \mathrm{~km} / \mathrm{s}$ is in the range of possible quantities for cometary impact at Venus orbit. Assuming asteroidal origin of the impactor with the density of $2.7 \mathrm{~g} / \mathrm{cm}^{3}$, the impact velocity of $>80 \mathrm{~km} / \mathrm{s}$ is estimated, and silicate materials are inappropriate for the origin of the impactor for this crater.

In the case of the crater in Figure $1 \mathrm{~b}, \mathrm{r}_{h l}$ and $\mathrm{r}_{h s}$ are approximately $98 \mathrm{~km}$ and $43 \mathrm{~km}$, respectively. From these ratios, an impact angle of $\sim 26^{\circ}$ is obtained. Assuming the impact of an icy body, the calculated impact velocity on the surface $V_{i}$ of $\sim 17 \mathrm{~km} / \mathrm{s}$ and $R$ of $\sim 1000 \mathrm{~m}$ are obtained. The corresponding incident velocity is $\sim 20 \mathrm{~km} / \mathrm{s}$. This is also plausible for Venus. In the case of the impact of an asteroidal object with the density of $2.7 \mathrm{~g} / \mathrm{cm}^{3}$, an impact velocity of $29 \mathrm{~km} / \mathrm{s}$ and an initial radius of the projectile of $580 \mathrm{~m}$ are required. Either type of the projectile is plausible for this crater, although the impact velocity is relatively smaller than the characteristic velocity of cometary impact, or higher than that of an asteroidal impact. The estimated impact angle is smaller than that of the projectile which induced the impact crater in Figure 1a. This agrees with the difference of the ejecta shapes in the two craters which show the impact crater in Figure $1 \mathrm{~b}$ formed from an impactor with a smaller impact angle. The shape of the halos in Figure 1b displays a butterfly shape in the up-range. This also agrees with the lower impact angle.

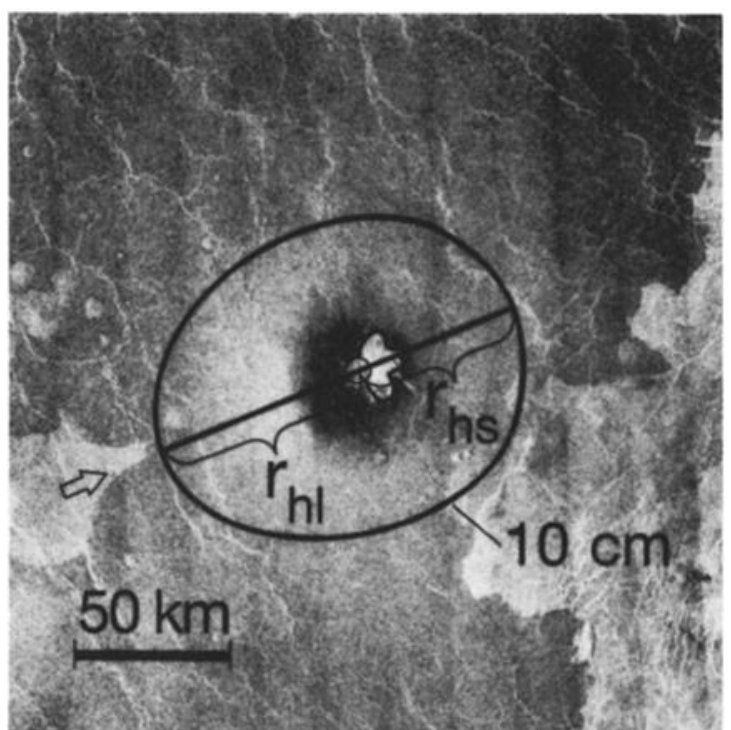

Figure 15. Crater image of Figure 1a with the contour that represents the calculated maximum saltation boulder size of $10 \mathrm{~cm}$ resulting from the impact of the meteoroid of a $450 \mathrm{~m}$ radius with an impact velocity of $45 \mathrm{~km} / \mathrm{s}$ and an impact angle of $43^{\circ}$. 
From the application to the above two impact craters, the bow shock model can, in principle, explain the formation of halos and their geometry.

\subsection{Constraints of the Bow Shock Model}

We assumed that the shape and mass of a meteoroid are constant while it travels in the Venusian atmosphere. We also assumed that the incoming angle is constant and the meteoroid does not decelerate in the Venusian atmosphere. However, the initial shape of the meteoroid may not be spherical. There may also be deformation, fragmentation, or ablation taking place in the atmosphere. The trajectory of the meteoroid may also change due to the atmospheric drag or to a change of mass. We discuss these effects on the bow shock model and constraints here.

The deformation or the irregular shape of the body does not affect the power law of (4) in the far wake if we use the characteristic radius $R_{n}$ (the radius of the curvature on the stagnation point) instead of the radius of the sphere $R$ [Martin, 1966]. For example, if the body is cylindrical with the radius of the cylinder $R_{c l}$ and the plane surface faces the flow field, (1) becomes $\Delta_{s} / R_{c l} \sim$ $[(\gamma-1) / 2]^{05}[\operatorname{Mart\imath n}, 1966]$, and $R_{n}$ approaches

$$
R_{n} \sim\left(\Delta_{s}^{2}+R_{c l}^{2}\right) /\left(2 \Delta_{s}\right)
$$

as $M \rightarrow \infty$, as the first approximation.

Fragmentation of meteoroids is expected to be a common phenomenon, since the strength of the stony meteorites is $\sim 6-500 \mathrm{MPa}[$ Melosh; 1981] and the dynamic pressure at the stagnation point $\rho V^{2}$ of $10^{2} \mathrm{MPa}$ is easily achieved at an altitude below $60 \mathrm{~km}$ for meteoroid velocities of $10 \mathrm{~km} / \mathrm{s}$. Meteoroid fragmentation was expected to decelerate a body. Also, dispersing of a meteoroid enlarges the area of the cross section of the object per unit mass, and as a result, the drag force acting on the unit mass of the body becomes larger. Passey and Melosh [1980] explained the formation of some terrestrial multiple craters analytically by applying their model of impacts of small objects fragmented by aero-breaking. They assumed that a single spherical body was brokenup into two spherical bodies within a single bow shock, and they separate with a certain lateral velocity. Pancake models [Melosh, 1981; Zahule, 1992] extended their work to describe that fragments spread over normal to the trajectory and the continuous object is flattened up to the width of a few tens times the original cross sectional area. However, neither of these analytical investigations takes into account the high pressure acting on the lateral sides of the fragment in a bow shock.

Moreover, recent numerical simulations [Takata et al., 1994; Crawford et al., 1995] show that the bow shock tends to contain a collection of fragments within a bow shock rather than dispersing the fragments, and the swarm of fragments itself changes its shape to minimize the atmospheric drag and deceleration in the high atmospheric pressure in the case of hypersonic impact. Experimental results [Schultz and Gault, 1992] also show that the extension of the lateral dispersion of a collection of small bodies largely depends on the atmospheric pressure. Therefore lateral spreading is also minimized, and deceleration due to the breakup would be smaller than the degree which has been previously considered.

In the classical ablation model [Bronshten, 1983], the change in the size of a meteoroid due to ablation is approximately expressed as $R_{i} / R_{\infty} \sim \exp \left(\sigma\left(V_{\imath}^{2}-V_{\infty}^{2}\right) / 6\right)$ [Nagasawa, 1981] , where $R_{\imath}$ is the radius of the meteoroid when it impacts the surface, $R_{\infty}$ is the initial radius of the meteoroid before it encounters the atmosphere, and $\sigma$ is the ablation coefficient which ranges in values between $\sim 10^{-2} \mathrm{~s}^{2} / \mathrm{km}^{2}\left[\right.$ Zahnle, 1992] and $\sim 10^{-3}$ $\mathrm{s}^{2} / \mathrm{km}^{2}$ [Takata et al., 1994]. If $R_{\infty}<100 \mathrm{~m}, R_{\imath} / R_{\infty}$ decreases dramatically.

We assumed a uniform atmosphere in these calculations. If the size of the body becomes comparable to or larger than the scale height of the atmosphere $(\sim 15 \mathrm{~km}$ for Venus), the area impacted by the bow shock wave becomes smaller compared with the radius of the meteoroid, and the ratio of the impact energy coupled with the atmosphere to the initial impact energy becomes small. This phenomenon agrees with the Magellan observational fact that halos can be observed around the craters of relatively small sizes [Schaber et al, 1992]. The original atmospheric shock features can be modified by the strong zonal westward winds that can transport the ejecta from crater with diameters larger than $15 \mathrm{~km}$ and distribute the deposit as a parabolic feature [Arvadson et al., 1991; Vervack and Melosh, 1992].

Therefore, to summarize, the size of craters which is subjected to the above analysis and bow slock model is less than the order of $10^{1} \mathrm{~km}$. The bow shock model is directly applicable to meteoroids in the range of the radius between $\sim 100 \mathrm{~m}$ and $\sim 1 \mathrm{~km}$ for the expected impact velocities at the orbit of Venus. In the case of the existence of significant deceleration or mass loss, the characteristic size of a meteoroid has to be applied.

\section{Conclusions}

We have investigated the origin of the dark and bright halos around the craters that are observed by Magellan imagery. A bow shock model is developed to explain the horizontal profile of gas shock properties on the Venusian surface originating from a meteoroidal impact as the most likely origin of the dark and bright halos. From the propagation of a paraboloidal bow shock front interacting with a planetary surface, we consiclered the shock propagation of an oblique impact. The strong horizontal particle velocity induced by the shock wave can, in principle, loft the surface materials. These can then be fragmented via mutual collisions. We estimated the saltation boulder size induced by the gas flow, and the magnitude of the effect of crushing of surface materials. The results show that a wide area can be affected by the surface disturbance by the strong gas flow (wind) induced by shock wave. The radial range of the disturbed area becomes $10-17 R_{c}$ in the case of $V_{3}=20$ $\mathrm{km} / \mathrm{s}$ and $R=1 \mathrm{~km}$. As a result, gas shock inducing 
the disturbance of the surface materials is likely to be the origin of the changes of the radar backscatter which give rise to both dark and bright halos. The dark halo is originated from the fine porous materials produced by the mutual collision of saltated rocks. The bright halo is probably a result of a rougher surface where surface is excavated and boulders are turned over, but they are not sufficiently pulverized via mutual collision. This process results in the deposit of blocky materials. The impact velocity, radius of the meteoroid, and radius of the propagation of the supersonic shock wave ( $\sim$ radius of the halos) are related to the crater sizes using the bow shock model.

\section{Appendix A: Axisymmetric Similarity Solution}

The gas is assumed to be a perfect gas with a constant ratio of specific heats, and the flow is assumed to expand cylindrically. The equations to characterize the motion of the gas are then the momentum equation,

$$
\frac{\partial v}{\partial t}+v \frac{\partial v}{\partial r}+\frac{1}{\rho} \frac{\partial p}{\partial r}=0
$$

the continuity equation,

$$
\frac{\partial \rho}{\partial t}+\frac{\partial(v \rho)}{\partial r}+\frac{\rho v}{r}=0
$$

and the energy equation assuming that the flow is adiabatic,

$$
\frac{\partial\left(p \rho^{-\gamma}\right)}{\partial t}+v \frac{\partial\left(p \rho^{-\gamma}\right)}{\partial r}=0
$$

[Hornung, 1967; Hayes and Probstern, 1966]. These are solved as a similarity solution, that is, the solution at time $t$ is similar to the solution at any other time. The shock properties, such as $p, \rho$, and $v$, are the functions of the lateral similarity variable, $r / r_{s}$, and the values at $r_{s}$ (Figure 2b).

\section{Appendix B: Conservation Equations for Oblique Shock}

The conservation equations, through an oblique shock wave relating the upstream (subscript 1) and downstream (subscript 2) of density $\rho$, pressure $P$, Mach number $M$, deflection angle $\theta$, and temperature $T$ are given by

$$
\begin{aligned}
\frac{\rho_{2}}{\rho_{1}} & =\frac{(\gamma+1) M_{1}^{2} \sin ^{2} \alpha}{2+(\gamma-1) M_{1}^{2} \sin ^{2} \alpha} \\
\frac{p_{2}}{p_{1}} & =\frac{2 \gamma M_{1}^{2} \sin ^{2} \alpha-(\gamma-1)}{(\gamma+1)} \\
M_{2}^{2} \sin ^{2}(\alpha-\theta) & =\frac{\gamma+1+(\gamma-1)\left(M_{1}^{2} \sin ^{2} \alpha-1\right)}{\gamma+1+2 \gamma\left(M_{1}^{2} \sin ^{2} \alpha-1\right)} \\
\tan \theta & =\tan \alpha \frac{M_{1}^{2} \cos ^{2} \alpha-\cot ^{2} \alpha}{0.5 M_{1}^{2}(\gamma+\cos 2 \alpha)+1}
\end{aligned}
$$

$$
\frac{T_{2}}{T_{1}}=\frac{p_{2}}{p_{1}} \frac{\rho_{1}}{\rho_{2}}
$$

[Hornung, 1986]. Here $\theta$ is the deflected angle of a shock-processed (downstream) gas flow to the pre- shocked (upstream) gas flow.

Equations (B1) - (B5) must be applied iteratively to both initial and reflected shocks to identify the corresponding shock reflection type.

Acknowledgments. We thank all the personnel associated with the Magellan mission for their efforts. We thank H. Hornung (Caltech) for valuable suggestions and comments which lead to the development of the present shock model. We thank J. D. O'Keefe (Caltech), B. A. Ivanov (Russian Academy of Science), and B. Sturtevant (Caltech), for their valuable suggestions and comments on the shock interactions with Venus. We also thank C. Elachi (JPL) for discussions on interpretation of the relationship of SAR data and surface properties, J. J. Plaut (JPL) for help on the SAR data analysis, C. Leff (JPL) for providing Magellan data; R. Herrick (LPI), J. W. Holt (Caltech), and K. A. Tryka (Caltech) for their help on data analysis, and G. E. Danielson for producing the crater pictures. We thank $R$. $M$. Schmidt and an anonymous reviewer for their constructive advice and criticism. Research was supported under NAG1953. Division of Geological and Planetary Sciences, California Institute of Technology contribution 5086 .

\section{References}

Arvidson, R. E., V. R. Baker, C. Elachi, R. S. Saunders, and J. A. Wood, Magellan: Initial analysis of Venus surface modification, Science, 252, 270-275, 1991

Bagnold, R. A., The Physics of Blown Sand and Desert Dunes, 265 pp., Methuen, New York, 1941.

Bagnold, R. A., The Physics of Sediment Transpont by Wind and Water, 359 pp., Am. Soc. of Civ. Eng., New York, 1988.

Brackett, R. A., and W. B. McKinnon, Cratering mechanics on Venus: Pressure enhancement by the atmospheric ocean, Geophys. Res. Lett., 19, 2115-2118, 1992.

Brode, H. L., Review of nuclear weapons effects, Annu. Rev. Nucl. Sci., 18, 153-202, 1968.

Bronshten, V. A., Physics of Meteoric Phenomena, 356 pp. D. Reidel, Norwell, Mass., 1983

Courant, R., and K. O. Friedrichs, Supersonic Flow and Shock Waves, 464 pp., Wiley-Interscience, New York, 1948 .

Crawford, D. A., M. B. Boslough, T. G. Trucano, and A. C. Robinson, The impact of periodic comet Shoemaker-Levy 9 on Jupiter, Int. J. Impact. Eng., in press, 1995.

Elachi, C., Introduction to the Physics and Techniques of Remote Sensing, 413 pp., John Wiley, New York, 1987.

Florenskiy, K. P., A. T. Bazilevskiy, G. A. Burba, O. V. Nikolayeva, A. A. Pronin, A. S. Selivanov, M. K. Narayeva A. S. Panfilov, and V. P. Chemodanov, Panorama of Venera 9 and 10 landing sites, in Venus, edited by D. M Hunten, L. Colin, T. M. Donahue, and V. I. Moroz, pp. 149-153, Univ. of Arizona Press, Tucson, 1983.

Gault, D. E., and J. A. Wedekind, Experimental studies of oblique impact, Proc. Lunar Planet. Sct. Conf., 9th, 3843-3875, 1978.

Greeley, R., J. D. Iversen, R. N. Leach, J. R. Marshall, B. R. White, and S. H. Williams, Windblown sand on Venus: 
Preliminary results of laboratory simulations, Icarus, 57, 112-124, 1984.

Hayes, W. D, and R. F. Probstein, Hypersonic Flow Theory I, 602 pp., Academic, San Diego, Calif., 1966.

Heilig, W., Some aspects of curved shock waves, in Proceedings 5th Mach Reflection Symposium, edited by G. W. Ullrich, 1985.

Hilsenrath, J., C. W. Beckett, W. S. Benedict, L. Fano, H. J. Masi, R. L. Nuttall, Y. S. Touloukian, and H. W. Woolley, Tables of thermal properties of gases, Nat. Bur. Stand. Circ., 564, 488 pp., 1955.

Hornung, H. G., Inviscid hypersonic flow over axisymmetric power-law bodies, Aero. Note 288, 10 pp., Aust. Def. Sci. Serv., Aero. Res. Lab., Melbourne, 1967.

Hornung, H., Regular and Mach reflection of shock waves, Annu. Rev. Fluid. Mech., 18, 35-58, 1986.

Hyon, J., Comments on Magellan MIDR CD-ROMs, USANASA-JPL-MG-0001 V2 (CD-ROM), Planet. Data Sys., 1991.

Ivanov, B. A., A. T Basilevsky, V. P. Kryuchkov, and I. M. Chernaya, Impact craters of Venus: Analysis of Venera 15 and 16 data, Proc. Lunar Planet. Sci. Conf. 16th, Part 2, J. Geophys. Res., 91 suppl., D413-D430, 1986.

Ivanov, B. A., I. V. Nemchinov, V. A. Svetsov, A. A. Provalov, V. M. Khazins, and R. J . Phillips, Impact craters on Venus: Physical and mechanical models, $J$. Geophys. Res., 97, 16167-16181, 1992.

Iversen, J. D., R. Greeley, and J. B. Pollack, Windblown dust on Earth, Mars, and Venus, J. Atmos. Sci, 33, 24252429, 1976.

Martin, J. J., Atmospheric Reentry, 264 pp., Prentice-Hall, Englewood Cliffs, N. J., 1966.

Melosh, H. J., Atmospheric breakup of terrestrial impactors, Proc. Lunar Planet. Sci. Conf., 12A, 29-35, 1981.

Melosh, H. J., Impact Cratering-A Geologıc Process, 245 pp., Oxford Univ. Press, New York, 1989.

Mizutani, H., Y. Takagi, and S. Kawakami, New scaling laws on impact fragmentation, Icarus, 87, 307-326, 1990.

Nagasawa, K., Ablation of meteorites from the viewpoint of the meteor observation (in Japanese), Monthly Earth, $3(9), 588-595,1981$.

O'Keefe, J. D., and T. J. Ahrens, Large scale impact on the earth with an atmosphere, (abstract), Lunar Planet. Sci. Conf., 19th, 887-888, 1988.

O'Keefe, J. D., and T. J. Ahrens, Dynamics of large scale impacts on Venus and Earth, (abstract), Lunar Planet. Sci. Conf., 24th, 1101-1102, 1993.

Passey, Q. R., and H. J. Melosh, Effects of atmospheric breakup on crater field formation, Icarus, 42, 211-233, 1980.

Phillips, R. J., R. E. Arvidson, J. M. Boyce, D. B. Campbell, J. E. Guest, G. G. Schaber, and L. A. Soderblom, Impact craters on Venus: Initial analysis from Magellan, Scrence, 252, 288-297, 1991.

Rosenblatt, M., Numerical simulations of thermally precursed airblast characteristics for HOB explosions, in Pro- ceedings, 5th Mach Reflection Symposium, edited by G. W. Ullrich, 1985.

Sakurai, A., Blast wave theory, in Basic Developments in Fluid Dynamics, edited by M. Holt, pp. 309-375, Academic, San Diego, Calif., 1968.

Schaber, G. G., R. G. Strom, H. J. Moore, L. A. Soderblom, R. L. Kirk, D. J. Chadwick, D. D. Dawson, L. R. Gaddis, J. M. Boyce, and J. Russell, Geology and distribution of impact craters on Venus: What are they telling us?, $J$. Geophys. Res., 97, 13257-13301, 1992.

Schmidt, R. M., Meteor crater: Energy of formation - implications of centrifuge scaling, Proc. Lunar Planet. Scu. Conf. 11th, 2099-2128, 1980.

Schmidt, R. M., Experiments to investigate atmospheric effects on crater size, (abstract), Proc. Lunar Planet. Sci. Conf., 23rd, 1221-1222, 1992.

Schultz, P. H., and D. E. Gault, On surviving atmospheric entry, (abstract), Proc. Lunar Planet. Sci. Conf., 23rd, 1235-1236, 1992.

Seiff, A., Thermal structure of the atmosphere of Venus, in Venus, edited by D. M. Hunten, L. Colin, T. M. Donahue, and V. I. Moroz, pp. 215-279, Univ. of Arizona Press, Tucson, 1983.

Takata, T., J.D. O'Keefe, T. J. Ahrens, and G. S. Orton, Comet Shoemaker-Levy 9: Impact on Jupiter and plume evolution, Icarus, 109, 3-19, 1994.

Tani, I., Fluid Dynamics (in Japanese), 268 pp., Iwanami, Tokyo, 1967.

Ulaby, F. T., Radar signatures of terrain: Useful monitors of renewable resources, Proc. IEEE, 70(12), 1410-1428, 1982.

Vervack, R.J., and H. J. Melosh, Wind interaction with falling ejecta: Origin of the parabolic features on Venus, Geophys. Res. Lett., 19, 525-528, 1992.

Vincenti, W. G., and C. H. Kruger, Introduction to Physical Gas Dynamics, p. 182, John Wiley, New York, 1965.

White, F. M., Viscous Fluid Flow, 725 pp., McGraw-Hill, New York, 1974.

Zahnle, K. J., Airburst origin of dark shadows on Venus, $J$. Geophys. Res., 97, 10,243-10,255, 1992.

Zel'dovich, Y. B. and Y. P. Raizer, Physıcs of Shock Waves and High-Temperature Hydrodynamic Phenomena, pp. 93101, Academic, San Diego, Calif., 1966.

T. J. Ahrens, Division of Geological and Planetary Sciences, California Institute of Technology, Pasadena, CA 91125.

R. J. Phillips, Department of Earth and Planetary Sciences, Washington University, St. Louis, MO 63130.

T. Takata, Geological Institute, University of Tokyo, Bunkyo, Tokyo, 113 JAPAN.

(Received November 25, 1991; revised August 10, 1995; accepted August 25, 1995.) 